\title{
Capacity Lower Bound for the Ising Perceptron
}

\author{
Jian Ding \\ University of Pennsylvania \\ Philadelphia, Pennsylvania, USA \\ dingjian@wharton.upenn.edu
}

\author{
Nike Sun \\ Massachusetts Institute of Technology \\ Cambridge, Massachusetts, USA \\ nsun@mit.edu
}

\begin{abstract}
We consider the Ising perceptron with gaussian disorder, which is equivalent to the discrete cube $\{-1,+1\}^{N}$ intersected by $M$ random half-spaces. The perceptron's capacity is the largest integer $M_{N}$ for which the intersection is nonempty. It is conjectured by Krauth and Mézard (1989) that the (random) ratio $M_{N} / N$ converges in probability to an explicit constant $\alpha_{\star} \doteq 0.83$. Kim and Roche (1998) proved the existence of a positive constant $\gamma$ such that $\gamma \leqslant M_{N} / N \leqslant 1-\gamma$ with high probability; see also Talagrand (1999). In this paper we show that the Krauth-Mézard conjecture $\alpha_{\star}$ is a lower bound with positive probability, under the condition that an explicit univariate function $\mathscr{S}_{\star}(\lambda)$ is maximized at $\lambda=0$. Our proof is an application of the second moment method to a certain slice of perceptron configurations, as selected by the so-called TAP (Thouless, Anderson, and Palmer, 1977) or AMP (approximate message passing) iteration, whose scaling limit has been characterized by Bayati and Montanari (2011) and Bolthausen (2012). For verifying the condition on $\mathscr{S}_{\star}(\lambda)$ we outline one approach, which is implemented in the current version using (nonrigorous) numerical integration packages. In a future version of this paper we intend to complete the verification by implementing a rigorous numerical method.
\end{abstract}

\section{CCS CONCEPTS}

- Theory of computation $\rightarrow$ Randomness, geometry and discrete structures; • Computing methodologies $\rightarrow$ Neural networks.

\section{KEYWORDS}

Ising perceptron, approximate message passing.

\section{ACM Reference Format:}

Jian Ding and Nike Sun. 2019. Capacity Lower Bound for the Ising Perceptron. In Proceedings of the 51st Annual ACM SIGACT Symposium on the Theory of Computing (STOC '19), June 23-26, 2019, Phoenix, AZ, USA. ACM, New York, NY, USA, 12 pages. https://doi.org/10.1145/3313276.3316383

\section{INTRODUCTION}

We consider the Ising perceptron under gaussian disorder, defined as follows. Let $\left(g_{\mu, i}\right)_{\mu \geqslant 1, i \geqslant 1}$ be an array of i.i.d. standard gaussian random variables (zero mean, unit variance). Fix any real number

Permission to make digital or hard copies of all or part of this work for personal or classroom use is granted without fee provided that copies are not made or distributed for profit or commercial advantage and that copies bear this notice and the full citation on the first page. Copyrights for components of this work owned by others than ACM must be honored. Abstracting with credit is permitted. To copy otherwise, or republish, to post on servers or to redistribute to lists, requires prior specific permission and/or a fee. Request permissions from permissions@acm.org.

STOC '19, fune 23-26, 2019, Phoenix, AZ, USA

(c) 2019 Association for Computing Machinery.

ACM ISBN 978-1-4503-6705-9/19/06 . .\$15.00

https://doi.org/10.1145/3313276.3316383 $\kappa$ (our main result is for $\kappa=0$ ). For any integer $N \geqslant 1$, we define $M_{N} \equiv M_{N}(\kappa)$ to be the largest integer $M$ such that

$$
\bigcap_{\mu=1}^{M}\left\{J \in\{-1,+1\}^{N}: \sum_{i=1}^{N} \frac{g_{\mu, i} J_{i}}{N^{1 / 2}} \geqslant \kappa\right\} \neq \varnothing .
$$

Krauth and Mézard [16] conjectured that as $N \uparrow \infty$ the (random) ratio $M_{N}(\kappa) / N$ converges (in probability) to an explicit constant $\alpha_{\star}(\kappa)$, which for $\kappa=0$ is roughly 0.83 . This was one of several works in the statistical physics literature analyzing various perceptron models via the "replica" or "cavity" heuristics $[11-13,17]$. In particular, in the variant where $J$ ranges not over $\{-1,+1\}^{N}$ but over the entire sphere of radius $N^{1 / 2}$, the analogous threshold was computed by Gardner and Derrida [13]. Another common variation is to take $g_{\mu, i} \in\{-1,+1\}$ to be i.i.d. symmetric random signs (Bernoulli disorder). The conjectured thresholds differ between the Ising [16] and spherical [13] models, but do not depend on whether the disorder is Bernoulli or gaussian. While the classical problem is under Bernoulli disorder, we have chosen to work under gaussian disorder to remove some technical difficulties.

For the spherical perceptron, very sharp rigorous results have been obtained, including a proof of the predicted threshold for all nonnegative $\kappa$ under Bernoulli disorder [20, 22]. For the Ising perceptron, much less has been proved. One can introduce a parameter $\beta \geqslant 0$ and define the associated positive-temperature partition function $Z_{\kappa, \beta}$ (see (7) below). The replica calculation extends to an explicit prediction $[13,16]$ for the limit of $N^{-1} \ln Z_{\kappa, \beta}$ as $N \uparrow \infty$ with $M / N \rightarrow \alpha$. This formula has been proved to be correct at sufficiently high temperature (small $\beta$ ) under Bernoulli disorder [25]. For the original model (1), corresponding to zero temperature or $\beta=\infty$, the best rigorous result to date is that for $\kappa=0$ there exists positive $\gamma$ such that $\gamma \leqslant M_{N} / N \leqslant 1-\gamma$ with high probability $[15,24]$ as $N \uparrow \infty$ (see also [23] for some work on general $\kappa$ ). Our main result is the following:

Theorem 1.1. Consider the Ising perceptron at $\kappa=0$ under gaussian disorder. Under Condition 1.2 (below), we have

$$
\liminf _{N \uparrow \infty} \mathbb{P}\left(\frac{M_{N}}{N} \geqslant \alpha\right)>0
$$

for any $\alpha<\alpha_{\star}$, where $\alpha_{\star}$ is the prediction of Krauth and Mézard [16], defined formally by Proposition 1.3.

Condition 1.2. The univariate function $\mathscr{S}_{\star}$ defined by (55) satisfies $\mathscr{S}_{\star}(\lambda)<0$ for all $\lambda \notin\{0,1\}$.

As noted above, the choice of gaussian disorder is a simplification of the Bernoulli disorder case which does not affect the predicted threshold. We expect that the arguments of $[15,24]$ can be easily modified to give $\gamma \leqslant M_{N} / N \leqslant 1-\gamma$ with high probability in the gaussian disorder setting, but would not recover the sharp threshold $\alpha_{\star}$. In the remainder of this abstract we describe the main ideas 
and outline the proof of Theorem 1.1. The full version of this paper is available online at https://arxiv.org/abs/1809.07742.

\subsection{The Krauth-Mézard Threshold}

We next give the formal characterization of the predicted threshold [16] for the Ising perceptron. We give the expressions for general $\kappa \in \mathbb{R}$, although we focus on the case $\kappa=0$. Write $\varphi$ for the standard gaussian density, and $\Psi$ for the complementary gaussian distribution function:

$$
\varphi(x) \equiv \frac{\exp \left(-x^{2} / 2\right)}{(2 \pi)^{1 / 2}}, \quad \Psi(x) \equiv \int_{x}^{\infty} \varphi(u) d u .
$$

For any $\xi \in \mathbb{R}$, a standard gaussian conditioned to be $\geqslant \xi$ has density $\varphi_{\xi}(x)$ and mean $\mathcal{E}(\xi)$ where

$$
\varphi_{\xi}(x) \equiv \frac{\varphi(x) 1\{x \geqslant \xi\}}{\Psi(\xi)}, \quad \mathcal{E}(\xi) \equiv \frac{\varphi(\xi)}{\Psi(\xi)} .
$$

Given a fixed threshold $\kappa \in \mathbb{R}$ and a parameter $q \in[0,1)$ we define

$$
F_{q}(x) \equiv \frac{\mathcal{E}}{(1-q)^{1 / 2}}\left(\frac{\kappa-x}{(1-q)^{1 / 2}}\right) .
$$

The Gardner free energy (or Gardner volume formula) is the functional of $(q, \psi) \in[0,1) \times[0, \infty)$ defined by

$$
\begin{aligned}
\mathscr{G}_{\kappa}(\alpha, q, \psi) \equiv- & \frac{\psi(1-q)}{2}+\int \ln \left(2 \operatorname{ch}\left(\psi^{1 / 2} z\right)\right) \varphi(z) d z \\
& +\alpha \int \ln \Psi\left(\frac{\kappa-q^{1 / 2} z}{(1-q)^{1 / 2}}\right) \varphi(z) d z .
\end{aligned}
$$

Given $\kappa$ and $\alpha$, the stationarity equations for $(q, \psi) \mapsto \mathscr{G}_{\kappa}(\alpha, q, \psi)$ lead to the definitions

$$
\begin{aligned}
P(\psi) & \equiv \int \operatorname{th}\left(\psi^{1 / 2} z\right)^{2} \varphi(z) d z, \\
R_{\kappa}(q, \alpha) & \equiv \alpha \int F_{q}\left(q^{1 / 2} z\right)^{2} \varphi(z) d z .
\end{aligned}
$$

For $\kappa=0$ we abbreviate

$$
\begin{gathered}
R(q, \alpha)=R_{0}(q, \alpha), \\
\mathscr{G}(\alpha, q, \psi) \equiv \mathscr{G}_{0}(\alpha, q, \psi) .
\end{gathered}
$$

Define the following numerical constants:

$$
\begin{aligned}
\alpha_{\mathrm{lb}} & \equiv 0.833078599, & \alpha_{\mathrm{ub}} & \equiv 0.833078600, \\
q_{\mathrm{lb}} & \equiv 0.56394907949, & \psi_{\mathrm{lb}} & \equiv 2.5763513100, \\
q_{\mathrm{l}, \mathrm{u}} & \equiv 0.56394907950 & \psi_{\mathrm{l}, \mathrm{u}} & \equiv 2.5763513103, \\
q_{\mathrm{u}, 1} & \equiv 0.56394908029, & \psi_{\mathrm{u}, 1} & \equiv 2.5763513221, \\
q_{\mathrm{ub}} & \equiv 0.56394908030, & \psi_{\mathrm{ub}} & \equiv 2.5763513224
\end{aligned}
$$

Note that $q_{\mathrm{lb}}<q_{\mathrm{l}, \mathrm{u}}<q_{\mathrm{u}, 1}<q_{\mathrm{ub}}$ and likewise for $\psi$. The following gives a formal characterization of the Krauth-Mézard threshold $\alpha_{\star}$ :

Proposition 1.3. For $\kappa=0$, let $\alpha_{\mathrm{lb}}, \alpha_{\mathrm{ub}}, q_{\mathrm{lb}}, q_{\mathrm{ub}}$ be as defined by (5). For any $\alpha \in\left(\alpha_{\mathrm{lb}}, \alpha_{\mathrm{ub}}\right)$, it holds that

$$
\sup _{q \in\left(q_{\mathrm{lb}}, q_{\mathrm{ub}}\right)}\left\{\frac{d P(R(q, \alpha))}{d q}\right\}<1,
$$

and there is a unique $q_{\star} \equiv q_{\star}(\alpha) \in\left(q_{\mathrm{lb}}, q_{\mathrm{ub}}\right)$ satisfying the fixedpoint equation $q_{\star}=P\left(R\left(q_{\star}, \alpha\right)\right)$. Let $\psi_{\star}(\alpha) \equiv R\left(q_{\star}(\alpha), \alpha\right)$. On the interval $\left(\alpha_{\mathrm{lb}}, \alpha_{\mathrm{ub}}\right)$ the function $\mathscr{G}_{\star}(\alpha) \equiv \mathscr{G}_{(}\left(\alpha, q_{\star}(\alpha), \psi_{\star}(\alpha)\right)$ is welldefined and strictly decreasing, with a unique root $\alpha_{\star} \in\left(\alpha_{\mathrm{lb}}, \alpha_{\mathrm{ub}}\right)$.

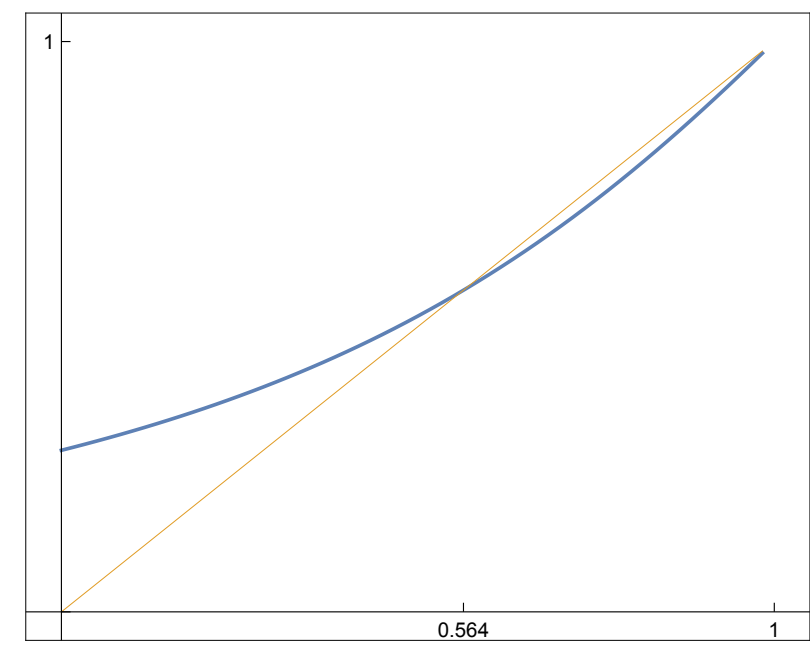

Figure 1: For $\alpha \doteq 0.833$ the function $q \mapsto P(R(q, \alpha))$ of (4), with fixed point $q_{\star}(\alpha) \doteq 0.564$. The figure also indicates that condition (6) is satisfied at $\alpha \doteq 0.833$, since the slope of the recursion at $q_{\star}$ is strictly less than one.

The map $q \mapsto P(R(q, \alpha))$ is shown in Figure 1 for $\alpha \doteq 0.833$. The function (3) is the conjectural free energy of the perceptron model. To be precise, for $\left(g_{\mu, i}\right)_{\mu \geqslant 1, i \geqslant 1}$ a doubly infinite array of i.i.d. standard gaussians, let $G_{M \times N}$ be the submatrix indexed by $1 \leqslant \mu \leqslant M$ and $1 \leqslant i \leqslant N$. The perceptron partition function associated to $G_{M \times N}$ at inverse temperature $\beta$ is given by

$$
Z_{\kappa, \beta} \equiv \sum_{J \in\{-1,+1\}^{N}} \exp \left\{\sum_{\mu=1}^{M}-\beta \mathbf{1}\left\{\sum_{i=1}^{N} \frac{g_{\mu, i} J_{i}}{N^{1 / 2}}<\kappa\right\}\right\} .
$$

We are interested in the zero-temperature limit $Z_{\kappa} \equiv Z_{\kappa, \infty}$; then $M_{N}(\kappa)$ is the largest $M$ for which $Z_{\kappa}\left(G_{M \times N}\right)>0$. The conjecture is that for all $\alpha \leqslant \alpha_{\star}(\kappa)$ there is a unique $q_{\star} \equiv q_{\star \kappa}(\alpha) \in[0,1)$ satisfying the fixed-point equation $q_{\star}=P\left(R_{\kappa}\left(q_{\star}, \alpha\right)\right)$, and

$$
\lim _{\substack{N \rightarrow \infty \\ M / N \rightarrow \alpha}} \frac{\ln Z\left(G_{M \times N}\right)}{N}=\mathscr{G}_{\star \kappa}(\alpha) \equiv \mathscr{G}_{\kappa}\left(\alpha, q_{\star \kappa}\left(\alpha, R_{\kappa}\left(q_{\star}, \alpha\right)\right)\right)
$$

where the limit holds in probability. The predicted limiting capacity $\lim _{N \rightarrow \infty} M_{N}(\kappa) / N=\alpha_{\star}(\kappa)$ is characterized by the relation $\mathscr{G}_{\star \kappa}\left(\alpha_{\star}(\kappa)\right)=0$. In fact there is an even stronger conjecture, the TAP approximation, which implies (8) and also guides our proof strategy. We explain this next.

\subsection{Moment Method and the TAP Free Energy}

We will prove Theorem 1.1 via the "second moment method" (or Paley-Zygmund inequality): for any nonnegative random variable $X$ having finite second moment,

$$
\frac{(\mathbb{E} X)^{2}}{\mathbb{E}\left(X^{2}\right)}=\frac{\mathbb{E}(X 1\{X>0\})^{2}}{\mathbb{E}\left(X^{2}\right)} \leqslant \frac{\mathbb{E}\left(X^{2}\right) \mathbb{P}(X>0)}{\mathbb{E}\left(X^{2}\right)}=\mathbb{P}(X>0), \text { (9) }
$$


where the intermediate bound follows from the Cauchy-Schwarz inequality. More generally, for any $\epsilon>0$,

$$
\frac{(\mathbb{E} X)^{2}(1-\epsilon)^{2}}{\mathbb{E}\left(X^{2}\right)} \leqslant \frac{(\mathbb{E} X 1\{X>\epsilon \mathbb{E} X\})^{2}}{\mathbb{E}\left(X^{2}\right)} \leqslant \mathbb{P}(X>\epsilon \mathbb{E} X) .
$$

Thus, if $\mathbb{E} X$ is large and $\mathbb{E}\left(X^{2}\right) \asymp(\mathbb{E} X)^{2}$ then we have $X \asymp \mathbb{E} X$ with positive probability.

It is important to note that the moment method cannot be directly applied to the perceptron partition function $Z_{\kappa}\left(G_{M \times N}\right)$. For the $\kappa=0$ case abbreviate $Z \equiv Z_{0}$. Trivially, its expected value is

$$
\mathbb{E} Z\left(G_{M \times N}\right)=\frac{2^{N}}{2^{M}}=\exp \{N(1-\alpha) \ln 2\} .
$$

However, there is a nontrivial regime $1-\gamma \leqslant \alpha \leqslant 1$ where it is proved [15] that $Z=0$ with high probability, even though $\mathbb{E} Z$ is exponentially large: in this regime $\mathbb{E}\left(Z^{2}\right)=(\mathbb{E} Z)^{2}$ certainly cannot hold since it would directly contradict (10). In fact it is conjectured that at any positive $\alpha$ the first moment $\mathbb{E} Z$ is much larger than the typical value $\exp \left\{N \mathscr{G}_{\star}(\alpha)\right\}$, and that as a consequence the relation $\mathbb{E}\left(Z^{2}\right) \asymp(\mathbb{E} Z)^{2}$ can hold at no positive $\alpha$.

To explain the fluctuations in $Z$, we take the TAP perspective [29]. The details are reviewed in the full version of the paper, but we summarize the key points here. Consider fixing $G_{M \times N}$ and sampling uniformly from the random subset

$$
\left\{J \in\{-1,+1\}^{N}: \frac{G_{M \times N} J}{N^{1 / 2}} \geqslant \kappa\right\} \subseteq\{-1,+1\}^{N} .
$$

Let $\mathbf{m}$ denote the mean value ("magnetization") of such a sample $J$; so $\mathbf{m}$ is a vector in $\mathbb{R}^{N}$ depending on $G$. Given $\mathbf{m}$ let

$$
q(\mathbf{m}) \equiv \frac{\|\mathbf{m}\|^{2}}{N}
$$

and let $\mathbf{h}(\mathbf{m}) \in \mathbb{R}^{M}$ be the unique solution to

$$
\mathbf{h}+(1-q(\mathbf{m})) F_{q(\mathbf{m})}(\mathbf{h})=\frac{G \mathbf{m}}{N^{1 / 2}} .
$$

(Existence of $\mathbf{h}$ turns out to be a nontrivial issue which will be discussed in Section 2; we assume it for now.) Define

$$
\psi(\mathbf{m}) \equiv \frac{\left\|F_{q(\mathbf{m})}(\mathbf{h})\right\|^{2}}{N} .
$$

The TAP free energy is the functional

$$
\begin{aligned}
\Phi^{\mathrm{TAP}}(\mathbf{m}) \equiv \sum_{i=1}^{N} \mathcal{H}\left(\frac{1+m_{i}}{2}\right)+\sum_{\mu=1}^{M} \ln \Psi\left(\frac{\kappa-h_{\mu}(\mathbf{m})}{(1-q(\mathbf{m}))^{1 / 2}}\right) \\
+\frac{N \psi(\mathbf{m})(1-q(\mathbf{m}))}{2} .
\end{aligned}
$$

Conjecturally, for $\alpha<\alpha_{\star}(\kappa)$, the vector $\mathbf{m}$ is the unique solution of the stationary equations for $\Phi^{\mathrm{TAP}}$.

Most importantly for us, it is conjectured that the sample fluctuations of this model, as induced by the randomness of the disorder $G$, are almost entirely captured by the vector $\mathbf{m}$, in that, with high probability over $G$, we have

$$
Z=\exp \left\{\Phi^{\mathrm{TAP}}(\mathbf{m})+O(1)\right\} .
$$

At the same time it is expected that $\mathbf{H} \equiv$ ath $\mathbf{m}$ "resembles" a vector of i.i.d. samples from the centered gaussian law with variance $\psi_{\star}(\alpha)$. This implies $q(\mathbf{m}) \doteq q_{\star}(\alpha)$ and $\psi(\mathbf{m}) \doteq \psi_{\star}(\alpha)$. Thus (12) is a much stronger conjecture than $(8)$ since $N^{-1} \Phi^{\mathrm{TAP}}(\mathbf{m}) \rightarrow \mathscr{G}_{\star \kappa}(\alpha)$. It also suggests one likely explanation for the failure of the basic moment method in this model: if $\mathbf{m}$ has gaussian fluctuations as expected, then the functional form of (11) would suggest that $\Phi^{\mathrm{TAP}}(\mathbf{m})$ fluctuates like a gaussian of variance $N c^{2}$ about its mean $\mathbb{E} \Phi^{\mathrm{TAP}}(\mathbf{m}) \doteq N \mathscr{G}_{\star K}(\alpha)$. As a result the first moment

$$
\mathbb{E} Z \doteq \exp \left\{\mathbb{E} \Phi^{\mathrm{TAP}}(\mathbf{m})+\frac{N c^{2}}{2}\right\}
$$

is much larger than the conjectured typical value for $Z$, and the second moment

$$
\mathbb{E}\left(Z^{2}\right) \doteq \exp \left\{2 \mathbb{E} \Phi^{\mathrm{TAP}}(\mathbf{m})+2 N c^{2}\right\}
$$

is much larger than the first moment squared.

The TAP heuristic suggests a natural proof strategy, which is to apply the moment method conditional on the marginal statistics $(\mathbf{m}, \mathbf{h}(\mathbf{m})) \in \mathbb{R}^{N} \times \mathbb{R}^{M}$. Indeed, for fixed $\mathbf{m}$ and $\mathbf{h}$, the characterization of $\mathbf{m}$ as a stationary point of $\Phi^{\mathrm{TAP}}$ can be expressed as a collection of linear constraints on $G$ (see below). It suggests at first glance that the conditional moments may reduce to tractable computations on linearly constrained gaussians. The problem with this approach is that the stationary points $\mathbf{m}$ are of course correlated with $G$, and we do not know if there is a unique one, although it is conjectured to be the case. In variants of the SherringtonKirkpatrick (SK) model the critical points of the TAP free energy have been more successfully studied [8], but the computation for SK is very difficult and we do not have an extension to the perceptron model.

\subsection{An Iterative Approach}

In this paper, we instead follow a constructive approach [5] of iterating the TAP stationary equations from a deterministic starting point $\mathbf{m}^{(1)}=q^{1 / 2} \mathbf{1}$. This has the advantage that after $t$ iterations we have explicit control on dependencies among $G, \mathbf{m}^{(1)}, \ldots, \mathbf{m}^{(t)}$. Moreover, the analysis of $[3,5]$ gives convergence to stationarity in the sense that

$$
\lim _{t \uparrow \infty} \limsup _{N \uparrow \infty} \frac{\left\|\mathbf{m}^{(t+1)}-\mathbf{m}^{(t)}\right\|^{2}}{N}=0 .
$$

At a high level this iterative approach is related to ideas of $[4,7]$. In our setting, we conjecture that in the limit of large $t$, the $\sigma$-field $\mathscr{F}_{t} \equiv \sigma\left(\mathbf{m}^{(1)}, \ldots, \mathbf{m}^{(t)}\right)$ captures almost all of the fluctuations of $Z$. More precisely, we conjecture that for some error $\lim _{t \uparrow \infty} c_{t}=0$, it holds for all $N$ large enough that

$$
\exp \left\{-N c_{t}\right\} \leqslant \frac{\mathbb{E}\left(Z \mid \mathscr{F}_{t}\right)}{\exp \left\{N \mathscr{G}_{\star}(\alpha)\right\}} \leqslant \exp \left\{N c_{t}\right\},
$$

(i.e., the first moment matches the prediction) and moreover that

$$
\frac{\mathbb{E}\left(Z^{2} \mid \mathscr{F}_{t}\right)}{\mathbb{E}\left(Z \mid \mathscr{F}_{t}\right)^{2}} \leqslant \exp \left\{N c_{t}\right\} .
$$

We expect the last bound to be accurate, in that the ratio between $\mathbb{E}\left(Z^{2} \mid \mathscr{F}_{t}\right)$ and $\mathbb{E}\left(Z \mid \mathscr{F}_{t}\right)^{2}$ should in fact grow exponentially in $N$ for any fixed $t$. For this reason, the second moment inequality (9) also cannot be directly applied to the random variables $\mathscr{F} t$. Nevertheless, this is a good approximation of the overall proof idea. The 
principal challenge in this paper was to devise a modified conditioning scheme together with a suitably truncated random variable $Z \leqslant Z$ such that (13) holds with an $O(1)$ rather than $\exp \left\{N c_{t}\right\}$ upper bound.

We conclude this section with a formal (and somewhat abstract) statement of our second moment result. The concrete construction is given in Section 2. In the proof we will introduce parameters $\delta, t, \epsilon$ where $\delta, \epsilon$ are positive constants and $t$ is a positive integer. We write $\eta$ for an error term depending on $M, N, \delta, t, \epsilon$ such that

$$
\underset{\delta \downarrow 0}{\limsup } \limsup _{t \uparrow \infty} \limsup _{\epsilon \downarrow 0} \limsup _{N \uparrow \infty,}\left|\eta_{M, N, \delta, t, \epsilon}\right|=0 \text {. }
$$

If a bound has multiple distinct error terms we indicate this by writing $\eta, \dot{\eta}$, etc.

THEOREM 1.4. Take the Ising perceptron at $\kappa=0$ under gaussian disorder, with partition function $Z \equiv Z\left(G_{M \times N}\right)$. We can define a collection of (integer) random variables $Z_{\delta, t, \epsilon} \equiv Z_{\delta, t, \epsilon}\left(G_{M \times N}\right)$, and a $\sigma$-field $\mathscr{F} \equiv \mathscr{F}_{M, N, \delta, t}$, such that the following hold: (i) with probability one $0 \leqslant Z_{\delta, t, \epsilon} \leqslant Z$; (ii) we have

$$
\liminf _{\delta \downarrow 0} \liminf _{t \uparrow \infty} \liminf _{\substack{N \uparrow \infty, M / N \rightarrow \alpha}} \mathbb{P}\left(\frac{\mathbb{E}\left(Z_{\delta, t, \epsilon} \mid \mathscr{F}\right)}{\exp \left\{N\left(\mathscr{G}_{\star}(\alpha)-\eta\right)\right\}} \geqslant 1\right)>0
$$

for all $\alpha \in\left(\alpha_{\mathrm{lb}}, \alpha_{\mathrm{ub}}\right)$; and (iii) assuming Condition 1.2, there exists $\alpha^{\prime} \in\left(\alpha_{\mathrm{lb}}, \alpha_{\star}\right)$ such that for all $\alpha \in\left(\alpha^{\prime}, \alpha_{\star}\right)$ we have

$$
\begin{aligned}
& \mathbb{E}\left(\left(Z_{\delta, t, \epsilon}\right)^{2} \mid \mathscr{F}\right) \leqslant \operatorname{cst}\left\{\mathbb{E}\left(Z_{\delta, t, \epsilon} \mid \mathscr{F}\right)\right\}^{2} \\
& \quad+\left(\exp \left\{2 N \mathscr{G}_{\star}(\alpha)-\frac{N}{\mathrm{cst}}\right\}+\exp \left\{N \mathscr{G}_{\star}(\alpha)\right\}\right) e^{N \dot{\eta}}
\end{aligned}
$$

where the $\mathrm{cst} \equiv \operatorname{cst}_{M, N, \delta, t, \epsilon}$ denote $\mathscr{F}_{M, N, \delta, t}$-measurable random variables that stay stochastically bounded in the limit $N \uparrow \infty$ with $M / N \rightarrow \alpha$.

The chief innovation of this paper is the design of the conditioning $\sigma$-field $\mathscr{F}$ and the random variables $Z_{\delta, t, \epsilon}$, given in Section 2 (with $Z_{\delta, t, \epsilon}$ defined explicitly by (37)). The main technical work is in the conditional moment analysis for $Z_{\delta, t, \epsilon}$, which occupies most of the full version of the paper. We will see that $Z_{\delta, t, \epsilon}\left(G_{M \times N}\right)$ in fact depends on $G_{M \times N}$ together with an extra small random perturbation $\boldsymbol{\kappa}^{\Delta}$ (appearing in (26)). The role of $\boldsymbol{\kappa}^{\Delta}$ is purely technical: it smooths a certain distribution and can only decrease the partition function, so it has no effect on the main result. We include $\boldsymbol{\kappa}^{\Delta}$ in the definition of $\mathscr{F}$, but otherwise will often suppress it from the notation.

Proof of Theorem 1.1 assuming Theorem 1.4. From the definition, we have $M_{N} \geqslant N \alpha$ if and only if $Z\left(G_{\lfloor N \alpha\rfloor \times N}\right)$ is positive, so

$$
\begin{aligned}
\mathbb{P}\left(\frac{M_{N}}{N} \geqslant \alpha \mid \mathscr{F}\right) & =\mathbb{P}\left(Z\left(G_{\lfloor N \alpha\rfloor \times N}\right)>0 \mid \mathscr{F}\right) \\
& \geqslant \mathbb{P}\left(Z_{\delta, t, \epsilon}\left(G_{\lfloor N \alpha\rfloor \times N}\right)>0 \mid \mathscr{F}\right)
\end{aligned}
$$

Abbreviate $Z_{\delta, t, \epsilon} \equiv Z_{\delta, t, \epsilon}\left(G_{\lfloor N \alpha\rfloor \times N}\right)$. For $\alpha^{\prime}<\alpha<\alpha_{\star}$ we have $\mathscr{G}_{\star}(\alpha)>0$, so we can choose $\delta, t, \epsilon$ such that

$$
\liminf _{\substack{N \uparrow \infty, M / N \rightarrow \alpha}}\left\{\min \left\{\mathscr{G}_{\star}(\alpha), \frac{1}{\mathrm{cst}}\right\}-2 \eta-\dot{\eta}\right\}>0
$$

for $\eta$ as in (15) and $\dot{\eta}$ as in (16) (both depending on $M, N, \delta, t, \epsilon$ ). Define the event

$$
E_{\eta} \equiv\left\{\frac{\mathbb{E}\left(Z_{\delta, t, \epsilon} \mid \mathscr{F}\right)}{\exp \left\{N\left(\mathscr{G}_{\star}(\alpha)-\eta\right)\right\}} \geqslant 1\right\} .
$$

On this event, combining (9) and (16) gives

$$
\frac{1}{\mathbb{P}\left(Z_{\delta, t, \epsilon}>0 \mid \mathscr{F}\right)} \leqslant \operatorname{cst}+\frac{e^{2 N \eta} e^{N \dot{\eta}}}{e^{N / \operatorname{cst}}}+\frac{e^{2 N \eta} e^{N \dot{\eta}}}{e^{N^{G_{\star}}(\alpha)}} .
$$

It follows that

$$
\mathbb{P}\left(Z_{\delta, t, \epsilon}>0 \mid \mathscr{F}\right) \geqslant \frac{1}{\operatorname{cst}+o_{N}(1)}
$$

where the term $o_{N}(1)$ tends to zero in the limit $N \rightarrow \infty$, with $M / N \rightarrow \alpha$, for any choice of $\delta, t, \epsilon$ such that (17) holds. Combining with (15) gives

$$
\begin{gathered}
\liminf _{N \uparrow \infty} \mathbb{P}\left(\frac{M_{N}}{N} \geqslant \alpha\right) \geqslant \liminf _{N \uparrow \infty} \mathbb{P}\left(Z_{\delta, t, \epsilon}\left(G_{\lfloor N \alpha\rfloor \times N}\right)>0\right) \\
\geqslant \liminf _{N \uparrow \infty} \mathbb{E}\left(\frac{1\left\{E_{\eta}\right\}}{\operatorname{cst}+o_{N}(1)}\right)>0,
\end{gathered}
$$

concluding the proof.

The proof of Theorem 1.4 occupies essentially the entirety of this paper. In Section 2 we define the $\sigma$-fields $\mathscr{F}$ and the random variables $Z_{\delta, t, \epsilon}$, and give the proof outline which is then implemented in the remainder of the paper.

Remark 1.5. Although our main result is for $\kappa=0$, this assumption is used only in a few steps which will be explicitly indicated. Otherwise we write most steps of the proof for general $\kappa \in \mathbb{R}$, assuming only that we have a fixed point $q_{\star}=P\left(R_{\kappa}\left(q_{\star}, \alpha\right)\right)$ such that condition (6) is satisfied for a range $\left(q_{\mathrm{lb}}, q_{\mathrm{ub}}\right) \ni q_{\star}$. We sometimes indicate the dependence on $\kappa, \alpha$ by writing

$$
\begin{aligned}
q_{\star} & \equiv q_{\star \kappa}(\alpha), \\
\psi_{\star} & \equiv \psi_{\star \kappa}(\alpha) \equiv R_{\kappa}\left(q_{\star}, \alpha\right), \\
\mathscr{G}_{\star \kappa}(\alpha) & \equiv \mathscr{G}_{\kappa}\left(\alpha, q_{\star \kappa}(\alpha), \psi_{\star \kappa}(\alpha)\right) .
\end{aligned}
$$

Remark 1.6. A closely related conditional second moment approach is implemented in an independent work [6] to compute the free energy of the Sherrington-Kirkpatrick (SK) spin glass [21], with external field, at high temperature. For SK and some related spin-glass models, a very powerful framework has been extensively developed (see $[14,18,26-28]$ and refs. therein) that extends to the more difficult low-temperature regime; but the approach of [6] offers an appealing alternative at high temperature. We point out that [6] computes conditional moments of the unrestricted SK partition function, yielding tight lower and upper bounds, but again only at very high temperature. The main challenge of the current paper is to prove an analogous lower bound as [6], but at zero temperature where the second moment method tolerates much less error, and furthermore for a model with a more complicated (nonlinear) Hamiltonian. 
A matching upper bound to Theorem 1.1 remains for us the most natural and interesting open question. Beyond this, we refer to intriguing experimental investigations $[1,2]$ suggesting surprising algorithmic phenomena for investigation in the Ising perceptron. In another direction we refer to $[9,10]$ suggesting a range of interesting phenomena to be investigated for the spherical perceptron in the $\kappa<0$ (replica symmetry breaking) regime.

\section{TAP ITERATION AND CONDITIONING SCHEME}

In the proof of Theorem 1.4, a small fraction of the columns of $G$ play a special role, and will be fully revealed in the preliminary setup. The subsequent moment calculation (occupying most of the paper) is based on the randomness in the remaining majority of columns, which are only partially revealed in the preliminary phase. For this reason it is convenient to slightly adjust the notation: we recast $N$ as $N_{\text {all }}$, and instead use $N$ for the portion of $G$ that plays the main role in the second moment. To be precise, for the remainder of the paper we let $M=\left\lfloor N_{\text {all }} \alpha_{\text {all }}\right\rfloor$ and

$$
G=G_{M \times N_{\text {all }}}=\left(\begin{array}{ll}
E & \hat{E}
\end{array}\right)
$$

where $E$ is an $M \times N$ matrix, $\hat{E}$ is an $M \times \hat{N}$ matrix, and we assume $N_{\text {all }}=N+\hat{N}$ with $\hat{N} / N=\hat{\alpha}(\delta) \rightarrow 0$ as $\delta \downarrow 0$. We write

$$
J_{\text {all }} \equiv\left(\begin{array}{c}
J \\
\hat{J}
\end{array}\right) \in\{-1,+1\}^{N_{\text {all }}}
$$

where $J \in\{-1,+1\}^{N}$ and $\hat{J} \in\{-1,+1\}^{\hat{N}}$. We then also relabel $\kappa$ as $\kappa_{\text {all }}$, so that the perceptron partition function $Z_{\kappa_{\text {all }}}(G)$ counts elements $J_{\text {all }} \in\{-1,+1\}^{N_{\text {all }}}$ satisfying

$$
\frac{G J_{\text {all }}}{\left(N_{\text {all }}\right)^{1 / 2}}=\frac{E J+\hat{E} \hat{J}}{\left(N_{\text {all }}\right)^{1 / 2}} \geqslant \kappa_{\text {all }} .
$$

For most of the proof it will be more convenient to normalize by $N^{1 / 2}$ rather than $\left(N_{\text {all }}\right)^{1 / 2}$. We therefore let

$$
\begin{aligned}
& \alpha \equiv \frac{M}{N}=\frac{\alpha_{\text {all }}-o_{N}(1)}{1-\hat{\alpha}(\delta)}, \\
& \kappa \equiv \kappa_{\text {all }}\left(\frac{N_{\text {all }}}{N}\right)^{1 / 2}=\frac{\kappa_{\text {all }}}{(1-\hat{\alpha}(\delta))^{1 / 2}}
\end{aligned}
$$

(so for our main result $\kappa=\kappa_{\text {all }}=0$ ). In this section we formally define the $\sigma$-field $\mathscr{F}$ of Theorem 1.4, and prove some results in preparation for the second moment analysis.

\subsection{TAP Iteration and State Evolution}

As mentioned above, the conditioning $\sigma$-field $\mathscr{F}$ of Theorem 1.4 is based on the so-called TAP or AMP (approximate message passing) iteration, a constructive approach to finding a stationary point of (11). We review the construction now, following [5].

Remark 2.1. To restate Remark 1.5 in our new notation, we now assume that we have $q_{\star} \equiv q_{\star} \kappa_{\text {all }}\left(\alpha_{\text {all }}\right)$ satisfying the fixed-point equation $q_{\star}=P\left(R_{\kappa_{\text {all }}}\left(q_{\star}, \alpha_{\text {all }}\right)\right)$, such that for some open interval $\left(q_{\mathrm{lb}}, q_{\mathrm{ub}}\right) \ni q_{\star}$ we have

$$
\sup _{q \in\left(q_{\mathrm{lb}}, q_{\mathrm{ub}}\right)}\left\{\frac{d P\left(R_{\kappa_{\mathrm{all}}}\left(q, \alpha_{\mathrm{all}}\right)\right)}{d q}\right\}<1 .
$$

Write $\psi_{\star} \equiv \psi_{\star \kappa_{\text {all }}}\left(\alpha_{\text {all }}\right) \equiv R_{\kappa_{\text {all }}}\left(q_{\star}, \alpha_{\text {all }}\right)$. We will arrange (in Proposition 2.3) to have $\hat{N} / N=\hat{\alpha}(\delta)=o_{1 / \delta}(1)$, where we use $o_{1 / \delta}(1)$ to indicate a quantity that tends to zero in the limit $\delta \downarrow 0$. As a result, by continuity considerations, for all sufficiently small $\delta$ there will be a value $q_{1} \equiv q_{\star \kappa}(\alpha)=q_{\star}+o_{1 / \delta}(1)$ satisfying the equation $q_{1}=P\left(R_{\kappa}\left(q_{1}, \alpha\right)\right)$ together with

$$
\sup _{q \in\left(q_{\mathrm{lb}}, q_{\mathrm{ub}}\right)}\left\{\frac{d P\left(R_{\kappa}(q, \alpha)\right)}{d q}\right\}<1
$$

(cf. (6) and (20)). This assumption holds for the rest of the paper, even when not explicitly stated. Let $\psi_{0} \equiv R_{\kappa}\left(q_{1}, \alpha\right)$.

For $\ell \geqslant 1, \mathbf{x} \in \mathbb{R}^{\ell}$ and $f: \mathbb{R} \rightarrow \mathbb{R}$ we will write $f(\mathbf{x})$ for the vector obtained by coordinatewise application of $f$, that is,

$$
f(\mathbf{x}) \equiv\left(f\left(x_{i}\right)\right)_{i \leqslant \ell} \in \mathbb{R}^{\ell} .
$$

Let $F \equiv F_{q_{1}}$ as defined by (2). Initialize

$$
\begin{aligned}
\mathbf{n}^{(0)} & \equiv \mathbf{0} \in \mathbb{R}^{M}, \\
\mathbf{m}^{(1)} & \equiv\left(q_{1}\right)^{1 / 2} \mathbf{1} \in \mathbb{R}^{N} .
\end{aligned}
$$

Apply the TAP equations

$$
\begin{array}{r}
\mathbf{n}^{(s)} \equiv F\left(\mathbf{h}^{(s)}\right) \equiv F\left(\frac{\boldsymbol{E m}^{(s)}}{N^{1 / 2}}-b^{(s)} \mathbf{n}^{(s-1)}\right), \\
\mathbf{m}^{(s+1)} \equiv \operatorname{th}\left(\mathbf{H}^{(s+1)}\right) \equiv \operatorname{th}\left(\frac{\boldsymbol{E}^{\mathrm{t}} \mathbf{n}^{(s)}}{N^{1 / 2}}-d^{(s)} \mathbf{m}^{(s)}\right),
\end{array}
$$

where $b^{(s)}, d^{(s)}$ are defined by

$$
\begin{aligned}
& b^{(s)}=\frac{\left(\mathbf{1}, \mathrm{th}^{\prime}\left(\mathbf{H}^{(s)}\right)\right)}{N}=1-\frac{\left\|\mathbf{m}^{(s)}\right\|_{2}^{2}}{N}, \\
& d^{(s)}=\frac{\left(\mathbf{1}, F^{\prime}\left(\mathbf{h}^{(s)}\right)\right)}{N} .
\end{aligned}
$$

This defines a sequence $\mathbf{n}^{(1)}, \mathbf{m}^{(2)}, \mathbf{n}^{(2)}, \ldots, \mathbf{m}^{(t)}, \mathbf{n}^{(t)}$. Then for all $s \geqslant 1$ denote $q_{s} \equiv\left\|\mathbf{m}^{(s)}\right\|_{2}^{2} / N$ and $\psi_{s} \equiv\left\|\mathbf{n}^{(s)}\right\|_{2}^{2} / N$, and note that the above definition implies $b^{(s)}=1-q_{s}$. We hereafter abbreviate $\mathbf{m} \equiv \mathbf{m}^{(t)}, \mathbf{H} \equiv \mathbf{H}^{(t)}$, and $q . \equiv q_{t}$.

Remark 2.2. For a bounded number $t$ of iterations, the $N \uparrow \infty$ distributional limit of TAP has been rigorously characterized in terms of a "state evolution" recursion $[3,5]$. For a special case of AMP, finite-sample results were obtained more recently, allowing even for $t$ growing slowly with $N$ [19]. In this work we only require some results from the earlier works $[3,5]$, which we informally summarize as follows:

a. For large $N$ and large $t$ the vectors $\mathbf{n}^{(t)} \equiv F\left(\mathbf{h}^{(t)}\right)$ and $\mathbf{n}^{(t-1)}$ are close in $\ell^{2}$; likewise the vectors $\mathbf{m}^{(t)} \equiv \operatorname{th}\left(\mathbf{H}^{(t)}\right)$ and $\mathbf{m}^{(t-1)}$ are close in $\ell^{2}$.

b. For any $s$, the empirical profile of $\mathbf{h}^{(s)}$ resembles a gaussian distribution with variance $q_{1}$, while the empirical profile of $\mathbf{H}^{(s)}$ resembles a gaussian distribution with variance $\psi_{0}$, where $q_{1}, \psi_{0}$ are as defined by Remark 2.1 .

c. For any fixed $t$, the matrix of inner products among the vectors $\mathbf{h}^{(1)}, \ldots, \mathbf{h}^{(t)}$ converges to a nondegenerate limit as $N \uparrow \infty$, as does the matrix of inner products among $\mathbf{H}^{(1)}, \ldots, \mathbf{H}^{(t)}$.

The formal statements will be reviewed below as required.

With $G_{M \times N_{\text {all }}}$ decomposed as in (18), we first prove the following: 
Proposition 2.3. For any small positive $\delta$ there is a decomposition (18) with $\hat{N} / N=\hat{\alpha}(\delta) \leqslant \delta / \exp \left(1 / \delta^{2 / 3}\right)$, and a large enough constant $t(\delta)$, such that the following holds: if $\mathbf{m} \equiv \mathbf{m}^{(t)}$ is defined by $t$ iterations of the TAP equations on $E$ for $t \geqslant t(\delta)$, then with probability at least $1 / 10$ there exists $\hat{J} \in\{-1,+1\}^{\hat{N}}$ satisfying

$$
\frac{1}{N^{1 / 2}} G_{M \times N_{\text {all }}}\left(\begin{array}{c}
\mathbf{m} \\
\hat{J}
\end{array}\right)=\frac{\boldsymbol{E} \mathbf{m}+\hat{E} \hat{J}}{N^{1 / 2}} \geqslant\left(\kappa+2 \delta^{1 / 2}\right) \mathbf{1}
$$

coordinatewise.

The proof of Proposition 2.3 is an adaptation of the argument of [15], and is deferred to the full version of the paper. The purpose of the proposition is explained by the following lemma:

Lemma 2.4. For any $y>\kappa$ there is a unique solution $h \in \mathbb{R}$ to the equation $h+(1-q \cdot) F_{q \cdot}(h)=y$.

Proof. Consider the function

$$
\begin{aligned}
L_{q \cdot}(h) & \equiv h+\left(1-q_{\bullet}\right) F_{q \cdot}(h) \\
& =h+\left(1-q_{\bullet}\right)^{1 / 2} \mathcal{E}\left(\frac{\kappa-h}{\left(1-q_{\bullet}\right)^{1 / 2}}\right) \geqslant \kappa
\end{aligned}
$$

where the last inequality holds because $\mathcal{E}(x) \geqslant x$ for all $x \in \mathbb{R}$. Since $\mathcal{E}(x)$ is always positive, we have $L_{q .}(h)>h$, so in particular $L_{q .}(h) \rightarrow \infty$ as $h \rightarrow \infty$. On the other hand, as $x \rightarrow \infty$ we have $\mathcal{E}(x)-x=1 / x$, which implies that as $h \rightarrow-\infty$ we have $L_{q .}(h)-\kappa=1 /|h|$. Finally, since $\mathcal{E}^{\prime}(x) \in(0,1)$ for all $x \in \mathbb{R}$, we have $d L_{q .} / d h \in(0,1)$ for all $h \in \mathbb{R}$. It follows that $h \mapsto L_{q .}(h)$ is a strictly increasing map from $\mathbb{R}$ onto $(\kappa, \infty)$, so a unique solution to the equation $L_{q} .(h)=y$ exists provided $y>\kappa$.

Define $\hat{J}$. to be the lexicographically minimal element of the space $\{-1,+1\}^{\hat{N}}$ satisfying

$$
\frac{1}{N^{1 / 2}} G_{M \times N_{\text {all }}}\left(\begin{array}{c}
\mathbf{m} \\
\hat{J} \cdot
\end{array}\right)=\frac{E \mathbf{m}+\hat{\boldsymbol{E}} \hat{J} \cdot}{N^{1 / 2}} \geqslant\left(\kappa+2 \delta^{1 / 2}\right) \mathbf{1}
$$

coordinatewise. Let $\boldsymbol{\kappa}^{\Delta}$ be an independent random vector sampled uniformly from the cube $\left[0, \delta / \exp \left(1 / \delta^{2}\right)\right]^{M}$, and solve for $\mathbf{h} \in \mathbb{R}^{N}$ such that

$$
\frac{E \mathbf{m}+\hat{E} \hat{J} .}{N^{1 / 2}}-\boldsymbol{\kappa}^{\Delta}=\mathbf{h}+\left(1-q_{\bullet}\right) F_{q \cdot}(\mathbf{h}) .
$$

Note that (26) is equivalent to

$$
\mathbf{n} \equiv F_{q .}(\mathbf{h})=F_{q} \cdot\left(\frac{E \mathbf{m}+\hat{E} \hat{J}_{\bullet}}{N^{1 / 2}}-\boldsymbol{\kappa}^{\Delta}-\left(1-q_{\bullet}\right) \mathbf{n}\right) .
$$

We recognize (27) as the TAP equation (22) with a perturbation that will have an essential role in the proof. Let

$$
\mathbf{m}^{(t+1)} \equiv \operatorname{th}\left(\mathbf{H}^{(t+1)}\right) \equiv \operatorname{th}\left(\frac{E^{\mathrm{t}} \mathbf{n}}{N^{1 / 2}}-d^{(t)} \mathbf{m}\right) .
$$

We emphasize that the construction of $\mathbf{m}^{(t+1)}$ differs from that of the previous $\mathbf{m}^{(s)}$, since we have passed through the perturbed equation (27). We continue to denote $q_{s} \equiv\left\|\mathbf{m}^{(s)}\right\|_{2}^{2} / N$ and $q$. $\equiv q_{t}$, and we also let $\psi_{\bullet} \equiv\|\mathbf{n}\|_{2}^{2} / N$. The conditioning $\sigma$-field in Theorem 1.4 is then given explicitly by $\mathscr{F} \equiv \mathscr{F}_{M, N_{\text {all }}, \delta, t, \epsilon} \equiv \sigma($ DATA) where DATA $\equiv \operatorname{DATA}_{M, N_{\text {all }}, \delta, t}$ is defined by

$$
\left(\mathbf{n}^{(1)}, \mathbf{m}^{(2)}, \mathbf{n}^{(2)}, \ldots, \mathbf{m}^{(t)} \equiv \mathbf{m}, \mathbf{n}^{(t)}, \mathbf{m}^{(t+1)}, \hat{E}, \boldsymbol{\kappa}^{\Delta}\right) .
$$

Note that $\mathscr{F}$ is contained in $\sigma\left(G_{M \times N_{\text {all }}}, \boldsymbol{\kappa}^{\Delta}\right)$.
Remark 2.5. Throughout this paper we write cst $\equiv \operatorname{cst}_{M, N_{\text {all }}, \delta, t, \epsilon}$ to indicate a collection of $\mathscr{F}_{M, N_{\text {all }}, \delta, t}$-measurable random variables that remains stochastically bounded in the limit $N_{\text {all }} \uparrow \infty$ with $M / N_{\text {all }} \rightarrow \alpha$ for fixed $\delta, t, \epsilon$ : that is to say,

$$
\limsup _{\substack{N_{\text {all }} \uparrow \infty, M / N_{\text {all }} \rightarrow \alpha}} \mathbb{P}\left(\operatorname{cst}_{M, N_{\text {all }}, \delta, t, \epsilon} \geqslant C\right) \downarrow 0
$$

as $C \uparrow \infty$, for any $\alpha \in\left(\alpha_{\mathrm{lb}}, \alpha_{\mathrm{ub}}\right)$. In our usage, the value of cst may change from one occurrence to the next, as long as the stochastic boundedness is maintained. If a result depends on multiple choices of cst simultaneously we will indicate this by writing cst, cst ${ }^{\prime}$, and so on. To indicate dependence on any other parameter, say $\gamma$, we shall write $\operatorname{cst}_{\gamma} \equiv \operatorname{cst}_{M, N_{\text {all }}, \delta, t, \epsilon, \gamma}$.

\subsection{Restricted Partition Function}

We next make a convenient change of coordinates. Let $\left(\mathbf{r}^{(s)}\right)_{1 \leqslant s \leqslant t}$ be an orthonormal basis for $\operatorname{span}\left(\left(\mathbf{m}^{(s)}\right)_{1 \leqslant s \leqslant t-1}, \mathbf{m}\right)$ where we denote $\mathbf{m} \equiv \mathbf{m}^{(t)}$. Likewise let $\left(\mathbf{c}^{(s)}\right)_{1 \leqslant s \leqslant t}$ be an orthonormal basis for $\operatorname{span}\left(\left(\mathbf{n}^{(s)}\right)_{1 \leqslant s \leqslant t-1}, \mathbf{n}\right)$ where $\mathbf{n}$ is the solution of (26). From now on we specify

$$
\mathbf{r}^{(t)} \equiv \frac{\mathbf{m}}{\left(N q_{\bullet}\right)^{1 / 2}}, \quad \mathbf{c}^{(t)} \equiv \frac{\mathbf{n}}{\left(N \psi_{\bullet}\right)^{1 / 2}}
$$

Explicitly, define the $N \times t$ matrix

$$
\boldsymbol{M} \equiv\left(\begin{array}{llll}
\frac{\mathbf{m}}{(N q \cdot)^{1 / 2}} & \frac{\mathbf{m}^{(t-1)}}{\left(N q_{t-1}\right)^{1 / 2}} & \cdots & \frac{\mathbf{m}^{(1)}}{\left(N q_{1}\right)^{1 / 2}}
\end{array}\right),
$$

and apply the Gram-Schmidt procedure to obtain $M=R \Gamma$ where $R$ is $N \times t$ with $R^{\mathrm{t}} R=\mathrm{I}_{t \times t}$ while $\Gamma$ is the $t \times t$ matrix containing the change-of-basis coefficients. (In the usual notation of $Q R$ factorization, $R$ corresponds to "Q" while $\Gamma$ corresponds to "R.") It follows from [3, Lemma 1(a)] that as $N \uparrow \infty$ with $t$ fixed, we have $\Gamma$ converging to a constant matrix; and by [3, Lemma $1(\mathrm{~g})]$ the limiting matrix is invertible (cf. Remark 2.2c). The columns of $\boldsymbol{R}=\mathbf{M} \boldsymbol{\Gamma}^{-1}$ give the desired orthonormal basis $\left(\mathbf{r}^{(t)}, \ldots, \mathbf{r}^{(1)}\right)$. To define $\mathbf{c}^{(s)}$ we instead consider the $M \times t$ matrix

$$
\boldsymbol{N} \equiv\left(\begin{array}{llll}
\frac{\mathbf{n}}{\left(N \psi_{\bullet}\right)^{1 / 2}} & \frac{\mathbf{n}^{(t-1)}}{\left(N \psi_{t-1}\right)^{1 / 2}} & \cdots & \frac{\mathbf{n}^{(1)}}{\left(N \psi_{1}\right)^{1 / 2}}
\end{array}\right),
$$

and obtain the QR factorization $N=C \Xi$ where $C$ is $M \times t$ with $C^{t} C=\mathrm{I}_{t \times t}$ while $\Xi$ is the $t \times t$ change-of-basis matrix. Since $\mathbf{n}$ is obtained by (27) rather than by the TAP iteration, the result of [3] does not give convergence of $\Xi$ in the limit $N \uparrow \infty$. Nevertheless, it follows from the additional randomness introduced by $\boldsymbol{\kappa}^{\Delta}$ that $\Xi$ is bounded and nondegenerate, in the sense that all its eigenvalues are bounded away from zero and infinity in the limit $N \uparrow \infty$. We will assume this fact for now, deferring the formal proof to the full paper. Note that both sets of basis vectors, $\left(\mathbf{r}^{(s)}\right)_{1 \leqslant s \leqslant t}$ and $\left(\mathbf{c}^{(s)}\right)_{1 \leqslant s \leqslant t}$, are measurable with respect to the $\sigma$-field $\mathscr{F} \equiv \sigma($ DATA) as defined by (29).

We now define the restricted partition function $Z_{\delta, t, \epsilon}\left(G_{M \times N_{\text {all }}}\right)$ which lower bounds $Z_{\kappa_{\text {all }}}\left(G_{M \times N_{\text {all }}}\right)$. The general idea is to first restrict to a certain (affine) slice of the discrete cube selected by the TAP iteration, then to restrict the perceptron satisfiability condition (19) by imposing additional constraints on the vector $G J_{\text {all }}$. The details are as follows. Let $\delta>0$, and decompose $G_{M \times N_{\text {all }}}$ as in (18) with $\hat{N} / N=\hat{\alpha}(\delta)$. Let $t \geqslant t(\delta)$ as specified by Proposition 2.3. 
On the $M \times N$ matrix $\boldsymbol{E}$, run the TAP equations (22) and (23) for $t$ iterations; then define $\hat{J}$. to be the lexicographically minimal element of $\{-1,+1\}^{\hat{N}}$ satisfying (25). From now on we only consider spin configurations of the form $J_{\text {all }}=\left(J, \hat{J}_{\text {. }}\right)$. If there exists no $\hat{J}$. satisfying (25) then we simply set $Z_{\delta, t, \epsilon} \equiv 0$. We also restrict to elements $J \in\{-1,+1\}^{N}$ which "resemble samples from $\mathbf{m}$ " in the following manner:

Definition 2.6 (restrictions in discrete cube). Conditional on the $\sigma$-field $\mathscr{F}_{M, N_{\text {all }}, \delta, t}$, we define $\mathbb{H}_{M, N_{\text {all }}, \delta, t}$ to be the set of spin configurations $J \in\{-1,+1\}^{N}$ such that the orthogonal projection of $J$ onto the span of the vectors $\mathbf{m}^{(1)}, \ldots, \mathbf{m}^{(t)}, \mathbf{H}^{(2)}, \ldots, \mathbf{H}^{(t)}, \mathbf{H}^{(t+1)}$ is very close to $\mathbf{m}^{(t)} \equiv \mathbf{m}$. Formally, we fix a large positive absolute constant $C$ and say that $J \in \mathbb{H}_{M, N_{\text {all }}, \delta, t}$ if $\mathbf{j} \equiv J / N^{1 / 2}$ can be decomposed as

$$
\mathbf{j}=\left(q_{J, t}\right)^{1 / 2} \mathbf{r}^{(t)}+\sum_{s=1}^{t-1} \gamma_{J, s} \mathbf{r}^{(s)}+\left(1-q_{J}\right)^{1 / 2} \mathbf{v}
$$

such that $\left|\gamma_{J, s}\right| \leqslant C / N$ for all $1 \leqslant s \leqslant t-1 ;\left|q_{J}-q.\right| \leqslant C / N$; and $\mathbf{v}$ is a unit vector which is exactly orthogonal to the span of the vectors $\mathbf{m}^{(1)}, \ldots, \mathbf{m}^{(t)}$, also is nearly orthogonal to the span of the vectors $\mathbf{H}^{(2)}, \ldots, \mathbf{H}^{(t+1)}$ in the sense that

$$
\max \left\{\left|\left(\mathbf{v}, \mathbf{H}^{(1)}\right)\right|, \ldots,\left|\left(\mathbf{v}, \mathbf{H}^{(t+1)}\right)\right|\right\} \leqslant \frac{C}{N^{1 / 2}} .
$$

Assuming that $1 / \epsilon$ is a positive integer, we let $\mathbb{H}_{\epsilon} \equiv \mathbb{H}_{M, N_{\text {all }}, \delta, t, \epsilon}$ be the subset of $J \in \mathbb{H}_{M, N_{\text {all }}, \delta, t}$ which additionally satisfy that for each integer $1 \leqslant \ell \leqslant 1 / \epsilon$ we have

$$
\left|\frac{\#\left\{i:(\ell-1) \epsilon \leqslant m_{i} \leqslant \ell \epsilon, J_{i}=1\right\}}{\#\left\{i:(\ell-1) \epsilon \leqslant m_{i} \leqslant \ell \epsilon\right\}}-\ell \epsilon\right| \leqslant 2 \epsilon,
$$

where $m_{i}$ is the $i$-th entry of the vector $\mathbf{m}$ from (26). We shall abbreviate $\mathbb{H} \equiv \mathbb{H}_{M, N_{\text {all }}, \delta, t}$ and $\mathbb{H}_{\epsilon} \equiv \mathbb{H}_{M, N_{\text {all }}, \delta, t, \epsilon}$.

We then restrict the satisfiability event as follows:

Definition 2.7 (profile truncation). For $J \in\{-1,+1\}^{N}$ we define the basic satisfiability event as

$$
S_{J, \delta, t} \equiv\left\{\frac{E J+\hat{E} \hat{J} .}{N^{1 / 2}} \geqslant \kappa 1+\kappa^{\Delta}\right\} .
$$

(Note $S_{J, \delta, t}$ implies $G J_{\text {all }} /\left(N_{\text {all }}\right)^{1 / 2} \geqslant \kappa 1$.) We then define a more restricted event $S_{J, \delta, t, \epsilon} \subseteq S_{J, \delta, t}$ as follows. Let

$$
\begin{array}{r}
\xi \equiv \frac{\kappa \mathbf{1}-\mathbf{h}}{\left(1-q_{\bullet}\right)^{1 / 2}}, \\
\zeta^{J} \equiv \frac{\left(q_{J, t}\right)^{1 / 2}\left(1-q_{\bullet}\right) \mathbf{n}}{\left(q_{\bullet}\right)^{1 / 2}\left(1-q_{J}\right)^{1 / 2}},
\end{array}
$$

and $\boldsymbol{v} \equiv \boldsymbol{E} \mathbf{v}+\zeta^{J}$. Assuming that $1 / \epsilon$ is a positive integer, we now define $S_{J, \delta, t, \epsilon}$ to be the event that

(i) $S_{J, \delta, t}$ occurs;

(ii) The vector $v$ satisfies the empirical moment bound

$$
\frac{1}{N} \sum_{\mu=1}^{M}\left(v_{\mu}\right)^{20} \leqslant \frac{2}{N} \sum_{\mu=1}^{M} \int v^{20} \varphi_{\xi_{\mu}}(v) d v
$$

(iii) For each pair of integers $1 \leqslant \ell, \ell^{\prime} \leqslant 1 / \epsilon$ we have

$$
\left|\begin{array}{c}
\#\left\{\begin{array}{c}
\mu:(\ell-1) \epsilon \leqslant \Psi\left(h_{\mu} /(q \cdot)^{1 / 2}\right) \leqslant \ell \epsilon \\
\text { and }\left(\ell^{\prime}-1\right) \epsilon \leqslant \Psi\left(v_{\mu}\right) / \Psi\left(\xi_{\mu}\right) \leqslant \ell^{\prime} \epsilon
\end{array}\right\} \\
\#\left\{\mu:(\ell-1) \epsilon \leqslant \Psi\left(h_{\mu} /(q \cdot)^{1 / 2}\right) \leqslant \ell \epsilon\right\}
\end{array}\right| \leqslant \epsilon^{10} .
$$

(Informally speaking, we wish to restrict to the event that the empirical distribution of $(\mathbf{h}, \boldsymbol{v})$ is close to the measure on $\mathbb{R}^{2}$ specified by the density function

$$
\frac{1}{(q .)^{1 / 2}} \varphi\left(\frac{h}{(q \cdot)^{1 / 2}}\right) \varphi_{\xi_{q .,}}(v) .
$$

This is formalized by conditions (ii) and (iii).)

We will prove Theorem 1.4 for the restricted perceptron partition function (with $M=\left\lfloor N_{\text {all }} \alpha_{\text {all }}\right\rfloor$ )

$$
Z_{\delta, t, \epsilon} \equiv Z_{\delta, t, \epsilon}\left(G_{M \times N_{\text {all }}}\right) \equiv \sum_{J \in \mathbb{H}_{M, N_{\text {all }}, \delta, t, \epsilon}} 1\left\{S_{J, \delta, t, \epsilon}\right\},
$$

which satisfies $0 \leqslant Z_{\delta, t, \epsilon} \leqslant Z_{\kappa_{\text {all }}}\left(G_{M \times N_{\text {all }}}\right)$ and is integer-valued. We shall always take $\epsilon \downarrow 0$ followed by $t \uparrow \infty$ followed by $\delta \downarrow 0$ while keeping $\kappa_{\text {all }}$ and $\alpha_{\text {all }}$ fixed. For this reason we often suppress dependence on $\kappa_{\text {all }}$ and $\alpha_{\text {all }}$ in order to simplify the notation. We will also often abbreviate $S_{J} \equiv S_{J, \delta, t}$ and $S_{J, \epsilon} \equiv S_{J, \delta, t, \epsilon}$.

\subsection{Proof Strategy}

As above, fix $\kappa_{\text {all }}, \alpha_{\text {all }}, q_{\star} \equiv q_{\star \kappa_{\text {all }}}\left(\alpha_{\text {all }}\right), \psi_{\star} \equiv \psi_{\star \kappa_{\text {all }}}\left(\alpha_{\text {all }}\right)$. To compute the (conditional) second moment of (37), we take a second $K \in \mathbb{H}_{\epsilon}$, so that $\mathbf{k} \equiv K / N^{1 / 2}$ has an analogous decomposition as (33),

$$
\mathbf{k}=\left(q_{K, t}\right)^{1 / 2} \mathbf{r}^{(t)}+\sum_{s=1}^{t-1} \gamma_{K, s} \mathbf{r}^{(s)}+\left(1-q_{K}\right)^{1 / 2} \tilde{\mathbf{v}} .
$$

Define the overlap between $J$ and $K$ as $\lambda \equiv \lambda_{J, K} \equiv(\mathbf{v}, \tilde{\mathbf{v}})$. Let

$$
\begin{aligned}
\mathbf{w} & \equiv \frac{\tilde{\mathbf{v}}-\lambda \mathbf{v}}{\left(1-\lambda^{2}\right)^{1 / 2}}, \\
c_{\lambda} & \equiv \frac{1-\lambda}{\left(1-\lambda^{2}\right)^{1 / 2}}=\frac{(1-\lambda)^{1 / 2}}{(1+\lambda)^{1 / 2}} .
\end{aligned}
$$

In the full paper we show that there is a constant $\lambda_{\min } \in(-1,0)$, explicitly defined by (46), such that

$$
\min _{J, K \in \mathbb{H}_{\epsilon}}\left\{\lambda_{J, K}\right\} \geqslant \lambda_{\min }-\eta
$$

where $\eta$ is some error tending to zero in the manner of (14). For any $\lambda_{\min }-\eta \leqslant \lambda \leqslant 1$ we let

$$
\begin{aligned}
& \mathbb{H}(\lambda) \equiv \mathbb{H}_{M, N_{\text {all }}, \delta, t}(\lambda) \equiv\left\{(J, K) \in\left(\mathbb{H}_{M, N_{\text {all }}, \delta, t}\right)^{2}:\right. \\
& \left.\lambda-\frac{1}{N} \leqslant \lambda_{J, K} \leqslant \lambda+\frac{1}{N}\right\}, \\
& \mathbb{H}_{\epsilon}(\lambda) \equiv \mathbb{H}_{M, N_{\text {all }}, \delta, t, \epsilon}(\lambda) \equiv\left\{(J, K) \in\left(\mathbb{H}_{M, N_{\text {all }}, \delta, t, \epsilon}\right)^{2}:\right. \\
& \left.\lambda-\frac{1}{N} \leqslant \lambda_{J, K} \leqslant \lambda+\frac{1}{N}\right\},
\end{aligned}
$$

so $\{-1,+1\}^{2 N} \supseteq \mathbb{H}^{2} \supseteq \mathbb{H}(\lambda) \supseteq \mathbb{H}_{\epsilon}(\lambda)$. To prove Theorem 1.4, we first consider events $S_{J, \epsilon} \equiv S_{J, \delta, t, \epsilon}$ and $S_{K} \equiv S_{K, \delta, t}$ for a fixed 
pair $(J, K) \in \mathbb{H}(\lambda)$ (for this part of the calculation, the further restriction from $\mathbb{H}$ to $\mathbb{H}_{\epsilon}$ is not needed). We condition on a background $\sigma$-field $\mathscr{F}^{\text {bg }}$ as discussed in Remark 2.8, and hereafter suppress it from the notation, so that $\mathbb{P}(\cdot)$ means $\mathbb{P}\left(\cdot \mid \mathscr{F}^{\mathrm{bg}}\right)$. We will compute $\mathbb{P}\left(S_{J, \epsilon}\right)$ and prove an upper bound on $\mathbb{P}\left(S_{K} \mid S_{J, \epsilon}\right)$. We then compute $\# \mathbb{H}_{\epsilon}$ and prove an upper bound on $\# \mathbb{H}_{\epsilon}(\lambda)$ (at this point the restriction from $\mathbb{H}$ to $\mathbb{H}_{\epsilon}$ becomes important). We shall see the formula (3) arise from the limits

$$
\begin{aligned}
& \frac{\ln \# \mathbb{H}_{\epsilon}}{N} \longrightarrow \mathscr{H}_{\star} \equiv \mathscr{H}_{\star \kappa_{\text {all }}}\left(\alpha_{\text {all }}\right) \\
& \quad \equiv-\psi_{\star}\left(1-q_{\star}\right)+\int \ln \left(2 \operatorname{ch}\left(\psi_{\star}^{1 / 2} z\right)\right) \varphi(z) d z \\
& \frac{\ln \mathbb{P}\left(S_{J, \epsilon}\right)}{N} \longrightarrow \mathscr{P}_{\star} \equiv \mathscr{P}_{\star \kappa_{\text {all }}}\left(\alpha_{\text {all }}\right) \\
& \quad \equiv \frac{\psi_{\star}\left(1-q_{\star}\right)}{2}+\alpha_{\text {all }} \int \ln \Psi\left(\frac{\kappa_{\text {all }}-\left(q_{\star}\right)^{1 / 2} z}{\left(1-q_{\star}\right)^{1 / 2}}\right) \varphi(z) d z
\end{aligned}
$$

where convergence holds in the limit $N_{\text {all }} \uparrow \infty$ followed by $t \uparrow \infty$ followed by $\delta \downarrow 0$, for any fixed $\epsilon>0$. Combining gives the first moment estimate (15), since $\mathscr{G}_{\star}=\mathscr{H}_{\star}+\mathscr{P}_{\star}$. We will see below that the (restricted) first moment (15) is relatively straightforward, whereas the second moment bound (16) requires significantly more involved calculations. We will introduce here the function $\mathscr{S}_{\star}(\lambda)$, and leave most of its interpretation and discussion to later sections. Let $P_{H, D}$ be the probability distribution on $\{-1,+1\}^{2}$ given by

$$
P_{H, D}=\left.\frac{1}{4}\left(\begin{array}{cc}
(1+m)^{2}+D & 1-m^{2}-D \\
1-m^{2}-D & (1-m)^{2}+D
\end{array}\right)\right|_{m=\text { th } H} .
$$

(We assume $D$ is such that $P_{H, D}$ is a nonnegative measure.) Let $\Gamma(H, D)$ be the (Shannon) entropy of $P_{H, D}$, so $0 \leqslant \Gamma(H, D) \leqslant \ln 4$. For $H \in \mathbb{R}$ and $A \in(0, \infty)$ let

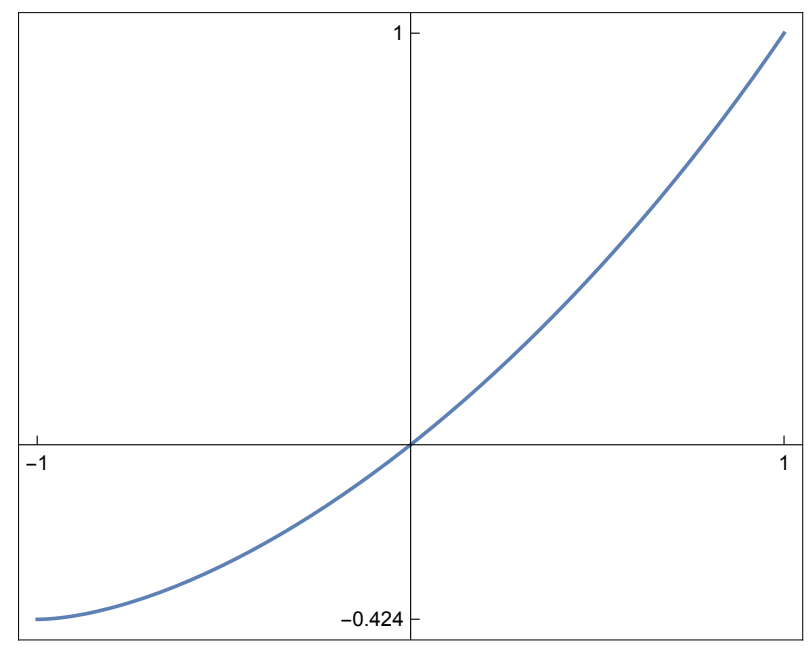

Figure 2: For $\alpha \doteq 0.833$, the $\operatorname{map} \tau \mapsto \ell(A(\tau))$ for $A(\tau) \equiv \exp (2 \operatorname{ath}(\tau))$ and $\ell$ as defined by (45). The map is strictly increasing, sandwiched between boundary values $\lambda_{\text {min }} \doteq-0.424$ (exact formula in (46)) and $\ell(\infty)=1$.

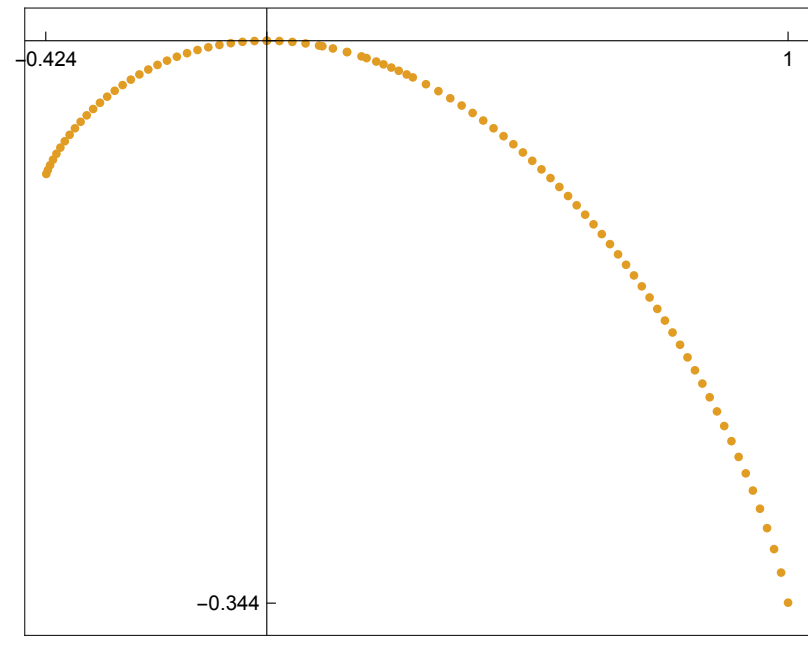

Figure 3: For $\alpha \doteq 0.833, \mathscr{H}(\lambda)$ as in (47). $\mathscr{H}(0)$ and $\mathscr{H}(1)=-\mathscr{H}_{\star} \doteq-0.344$.

$$
\left.D_{H}(A) \equiv \frac{\left(A^{2}-1\right)\left(1-m^{2}\right)^{2}}{\left(\left(A^{2}+m^{2}-(A m)^{2}\right)^{1 / 2}+1\right)^{2}}\right|_{m=\text { th } H} .
$$

We integrate over the distribution of $H$ to define

$$
\left.\ell(A) \equiv \int \frac{D_{\psi^{1 / 2} z}(A)}{1-q} \varphi(z) d z\right|_{q=q_{\star}, \psi=\psi_{\star}} .
$$

We show that the function $\ell$ is strictly increasing on $A \in(0, \infty)$, sandwiched by boundary values

$$
\begin{aligned}
\ell(0) & =-\int \frac{\left(1-\left|\operatorname{th}\left(\psi^{1 / 2} z\right)\right|\right)^{2}}{1-q} \varphi(z) d z \equiv \lambda_{\min } \in(-1,0), \\
\ell(\infty) & =\int \frac{1-\operatorname{th}\left(\psi^{1 / 2} z\right)^{2}}{1-q} \varphi(z) d z=1,
\end{aligned}
$$

where the last equality is by (4). See Figure 2 (where we chose a convenient parametrization $A=A(\tau) \equiv \exp (2 \operatorname{ath}(\tau)))$. The inverse $\lambda \mapsto \ell^{-1}(\lambda) \equiv A(\lambda)$ is well-defined for $\lambda_{\min } \leqslant \lambda \leqslant 1$. Let

$$
\mathscr{H}(\lambda) \equiv-2 \mathscr{H}_{\star}+\int \Gamma\left(\left(\psi_{\star}\right)^{1 / 2} z, D_{\left(\psi_{\star}\right)^{1 / 2} z}(A(\lambda))\right) \varphi(z) d z
$$

We will find that $\mathscr{H}(0)=0, \mathscr{H}(1)=-\mathscr{H}_{\star}$, and

$$
\underset{\delta \downarrow 0}{\limsup } \limsup _{t \uparrow \infty} \limsup _{\epsilon \downarrow 0} \limsup _{N_{\text {all } \uparrow \infty}} \frac{\ln \# \mathbb{H}_{\epsilon}(\lambda)}{N_{\text {all }}} \leqslant 2 \mathscr{H}_{\star}+\mathscr{H}(\lambda) \text {. }
$$

See Figure 3. Next let

$$
\xi_{q, z} \equiv \frac{\kappa_{\text {all }}-q^{1 / 2} z}{(1-q)^{1 / 2}},
$$

and note that $\xi_{q, z}$ appears in the above definition (42) of $\mathscr{P}_{\star}$. Recalling the definition of $\varphi_{\xi}$ from (2), let

$$
\begin{aligned}
\mathscr{I}_{s}(\lambda) \equiv \alpha_{\text {all }} \iint \ln \Psi & \left(\frac{\xi_{q, z}-\lambda v}{\left(1-\lambda^{2}\right)^{1 / 2}}-\frac{\mathcal{E}\left(\xi_{q, z}\right) \cdot s}{\psi^{1 / 2}(1-q)^{1 / 2}}\right) \\
& \times\left.\varphi_{\xi_{q, z}}(v) d v \varphi(z) d z\right|_{q=q_{\star}, \psi=\psi_{\star}}
\end{aligned}
$$




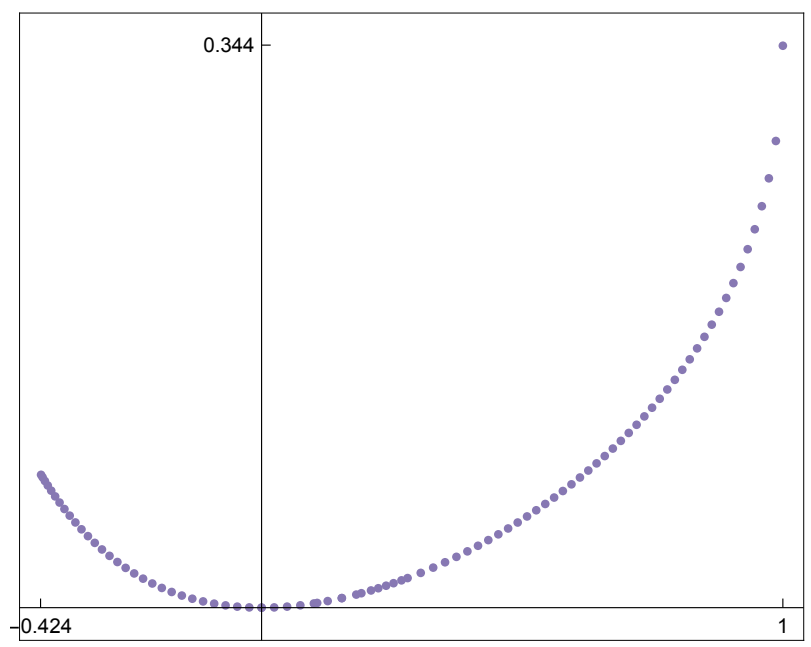

Figure 4: For $\alpha \doteq 0.833, \mathscr{P}(\lambda)$ as in (51). $\mathscr{P}(0)=0$ and $\mathscr{P}(1)=-\mathscr{P}_{\star} \doteq 0.344$

Abbreviate $\mathscr{I}(\lambda) \equiv \mathscr{I}_{0}(\lambda)$ and let

$$
\mathscr{P}(\lambda) \equiv-\mathscr{P}_{\star}+\frac{\psi_{\star}\left(1-q_{\star}\right)(1-\lambda)}{2(1+\lambda)}+\mathscr{I}(\lambda) .
$$

Then $\mathscr{P}(0)=0$ and $\mathscr{P}(1)=-\mathscr{P}_{\star}$; see Figure 4 . Let

$$
\begin{aligned}
\mathscr{B}(\lambda, s) \equiv \frac{s^{2}}{2} & +\mathscr{I}_{s}(\lambda)-\mathscr{I}(\lambda) \\
& \quad-\left(\psi_{\star}\right)^{1 / 2}\left(1-q_{\star}\right)^{1 / 2} s\left(\frac{1-\lambda}{1+\lambda}\right)^{1 / 2} .
\end{aligned}
$$

and define $\mathscr{A}(\lambda) \equiv \inf _{s} \mathscr{B}(\lambda, s)$; see Figure 5. We remark that for

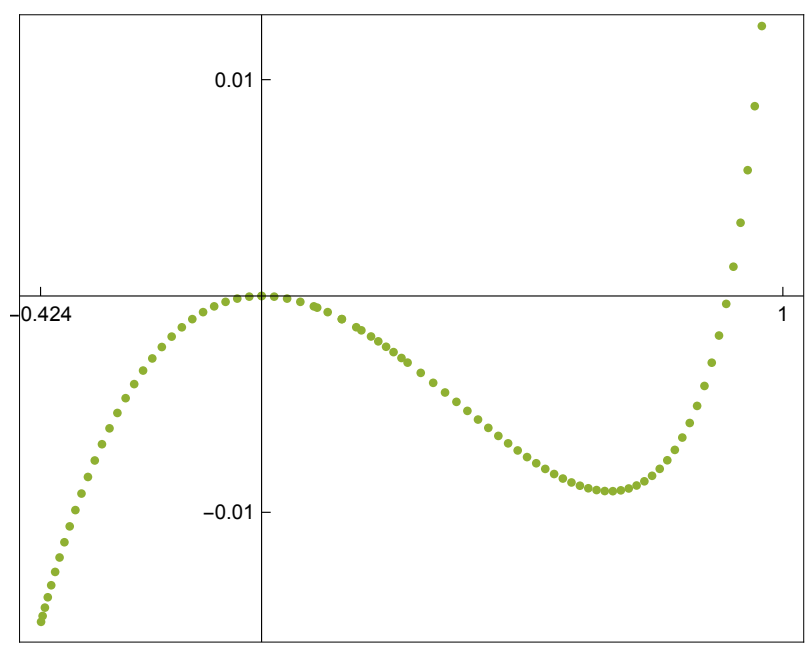

Figure 5: For $\alpha \doteq 0.833, \mathscr{B}(\lambda, s)$ from (52) with $s=-0.3 \lambda$.

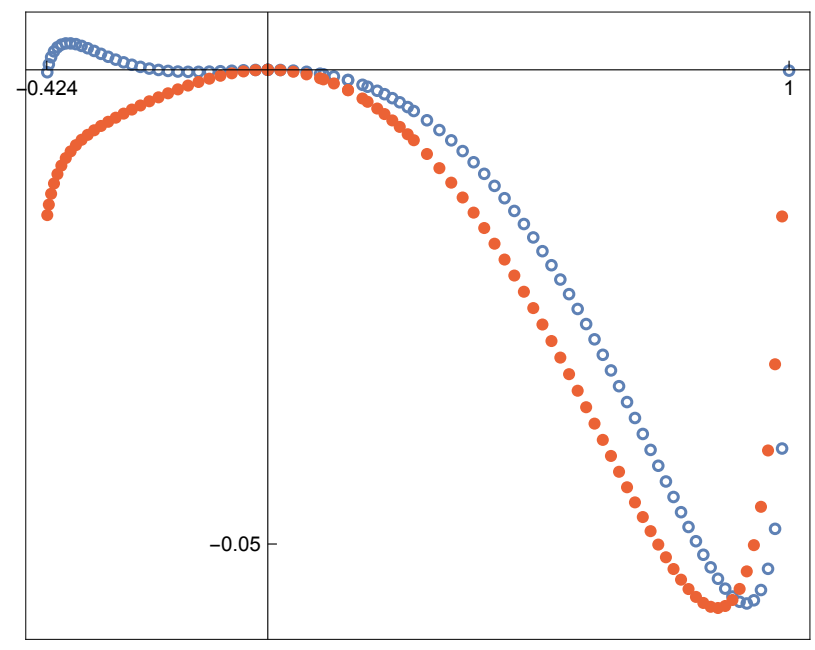

Figure 6: For $\alpha \doteq 0.833: \mathscr{H}(\lambda)+\mathscr{P}(\lambda)$ in blue open circles; $\mathscr{H}(\lambda)+\mathscr{P}(\lambda)+\mathscr{B}(\lambda,-0.3 \lambda)$ in red filled points.

all $\lambda$ we have $\mathscr{A}(\lambda) \leqslant \mathscr{B}(\lambda, 0)=0$, and

$$
\begin{aligned}
\left.\frac{\partial \mathscr{B}(0, s)}{\partial s}\right|_{s=0}=- & \left(\psi_{\star}\right)^{1 / 2}\left(1-q_{\star}\right)^{1 / 2} \\
& \quad+\alpha_{\text {all }} \int \frac{\mathcal{E}\left(\xi_{q_{\star}, z}\right)^{2}}{\psi_{\star}^{1 / 2}\left(1-q_{\star}\right)^{1 / 2}} \varphi(z) d z=0,
\end{aligned}
$$

where the last equality is by (4). The function $\mathscr{B}(\lambda, s)$ arises as a limit of cumulant-generating functions, and is therefore convex in $s$. It follows that $s=0$ is a minimizer of $\mathscr{B}(0, s)$, and so $\mathscr{A}(0)=0$. Since $\mathscr{A} \leqslant 0$ everywhere it follows that $\mathscr{A}^{\prime}(0)=0$ and $\mathscr{A}^{\prime \prime}(0) \leqslant 0$. We will find that

$$
\begin{array}{r}
\limsup \limsup _{\delta \downarrow 0} \limsup _{t \uparrow \infty} \limsup _{\epsilon \downarrow 0} \frac{\ln \mathbb{P}\left(S_{J, \epsilon}, S_{K}\right)}{N_{\text {all }} \uparrow \infty} \\
\leqslant 2 \mathscr{P}_{\star}+\mathscr{P}(\lambda)+\mathscr{A}(\lambda) .
\end{array}
$$

Let $\mathscr{S}(\lambda) \equiv \mathscr{H}(\lambda)+\mathscr{P}(\lambda)+\mathscr{A}(\lambda)$; see Figure 6. Although we have suppressed it from the above notation, we now recall that the functions $\mathscr{S}(\lambda), \mathscr{H}(\lambda), \mathscr{P}(\lambda), \mathscr{A}(\lambda)$ all depend on the parameters $\kappa_{\text {all }}$ and $\alpha_{\text {all }}$. We make the dependence explicit by writing $\mathscr{S}(\lambda) \equiv \mathscr{S}_{\kappa_{\text {all }}, \alpha_{\text {all }}}(\lambda)$ and so on. Then, from (48) and (53) it is easy to derive that

$$
\begin{aligned}
& \underset{\delta \downarrow 0}{\lim \sup } \limsup _{t \uparrow \infty} \limsup _{\epsilon \downarrow 0} \limsup _{N_{\text {all }} \uparrow \infty} \frac{\ln \mathbb{E}\left(Z_{\delta, t, \epsilon}\left(G_{\lfloor N \alpha\rfloor \times N}\right)^{2}\right)}{N_{\text {all }}} \\
& \leqslant 2 \mathscr{G}_{\star \kappa_{\text {all }}}\left(\alpha_{\text {all }}\right)+\mathscr{S}_{\kappa_{\text {all }}, \alpha_{\text {all }}}(\lambda)
\end{aligned}
$$

with $\mathscr{G}_{\star}=\mathscr{H}_{\star}+\mathscr{P}_{\star}$ as above. (The detailed derivation of (54) is given in the proof of Theorem 1.4.) Condition 1.2 refers to the 
function

$$
\begin{aligned}
\mathscr{S}_{\star}(\lambda) & \equiv \mathscr{S}_{0, \alpha_{\star}}(\lambda)=\left\{\frac{\psi(1-q)(1-\lambda)}{2(1+\lambda)}\right. \\
+ & \int \Gamma\left(\psi^{1 / 2} z, D_{\psi^{1 / 2} z}(A(\lambda))\right) \varphi(z) d z \\
+ & \inf _{s}\left[\frac{s^{2}}{2}-\psi^{1 / 2}(1-q)^{1 / 2} s\left(\frac{1-\lambda}{1+\lambda}\right)^{1 / 2}+\mathscr{I}_{s}(\lambda)\right] \\
& \left.-2 \mathscr{H}_{\star}-\mathscr{P}_{\star}\right\} \mid \begin{array}{c}
\kappa_{\text {all }}=0, \alpha_{\text {all }}=\alpha_{\star}, \\
q=q_{\star}\left(\alpha_{\star}\right), \psi=\psi_{\star}\left(\alpha_{\star}\right)
\end{array}
\end{aligned}
$$

for $\alpha_{\star}$ as given by Proposition 1.3. It is not difficult to see from (15) and (54) that Condition 1.2 is certainly a necessary condition for the result of Theorem 1.4.

Remark 2.8. We now make some comments on our conditioning scheme. We decompose $G_{M \times N_{\text {all }}}$ as in (18), and run the TAP equations (22) and (23) on $\boldsymbol{E}$ with deterministic initial starting vectors $\mathbf{n}^{(0)} \in \mathbb{R}^{M}$ and $\mathbf{m}^{(1)} \in \mathbb{R}^{N}$. For $s \geqslant 1$ we obtain $\mathbf{n}^{(s)}$ as a function of $\boldsymbol{E} \mathbf{m}^{(s)}$ and $\mathbf{n}^{(s-1)}$; then $\mathbf{m}^{(s+1)}$ as a function of $\boldsymbol{E}^{\mathrm{t}} \mathbf{n}^{(s)}$ and $\left.\mathbf{m}^{(s)}\right)$; and so on, up to $\mathbf{m}^{(t)} \equiv \mathbf{m}$ and $\mathbf{n}^{(t)}$. We define $\hat{J}$. in (25) as a measurable function of $\boldsymbol{E m}, \hat{E}$. We then sample an independent vector $\boldsymbol{\kappa}^{\Delta}$, and obtain $\mathbf{n}$ in (27) as a function of $\boldsymbol{E} \mathbf{m}, \hat{\boldsymbol{E}} \hat{J}$., and $\boldsymbol{\kappa}^{\Delta}$. Lastly we obtain $\mathbf{m}^{(t+1)}$ in (28) as a function of $\boldsymbol{E}^{\mathbf{t}} \mathbf{n}$ and $\mathbf{m}$. This defines for us $\mathscr{F}=\sigma($ DATA) with DATA as in (29). Conditional on $\mathscr{F}$, the matrix $E$ has the law of a standard gaussian in $\mathbb{R}^{M \times N}$ subject to the equations

$$
\begin{aligned}
\frac{\boldsymbol{E} \mathbf{m}^{(s)}}{N^{1 / 2}} & =\mathbf{h}^{(s)}+b^{(s)} \mathbf{n}^{(s-1)}, \\
\frac{\boldsymbol{E}^{\mathrm{t}} \mathbf{n}^{(s)}}{N^{1 / 2}} & =\mathbf{H}^{(s+1)}+d^{(s)} \mathbf{m}^{(s)}
\end{aligned}
$$

for $1 \leqslant s \leqslant t-1$, together with (see (27) and (28))

$$
\begin{aligned}
\frac{\boldsymbol{E} \mathbf{m}+\hat{E} \hat{J}_{\bullet}}{N^{1 / 2}}-\boldsymbol{\kappa}^{\Delta} & =\mathbf{h}+(1-q .) \mathbf{n}, \\
\frac{\boldsymbol{E}^{\mathrm{t}} \mathbf{n}}{N^{1 / 2}} & =\mathbf{H}^{(t+1)}+d^{(t)} \mathbf{m} .
\end{aligned}
$$

Let $\mathbb{Q}$ denote the marginal law of the sequence DATA. For the remainder of this paper, we first sample DATA $\sim \mathbb{Q}$, and make all calculations conditional on a background $\sigma$-field $\mathscr{F}$ bg $\equiv \sigma($ DATA $)$. We then let $E$ be a standard gaussian in $\mathbb{R}^{M \times N}$, independent of $\mathscr{F}$ bg until further notice. We shall subsequently reintroduce constraints on $E$ in a way that is equivalent to conditioning on $\mathscr{F}$.

\section{CHANGE OF MEASURE}

Let $\hat{\mathbf{e}}_{\mu}$ be the $\mu$-th coordinate vector in $\mathbb{R}^{M}$, and $\dot{\mathbf{e}}_{i}$ the $i$-th coordinate vector in $\mathbb{R}^{N}$. For any $\mathbf{x} \in \mathbb{R}^{M}$ let $X_{i}$ denote the $M \times N$ matrix with $\mathbf{x}$ in column $i$ and all other columns zero, $X_{i} \equiv \mathbf{x}\left(\dot{\mathbf{e}}_{i}\right)^{\mathrm{t}}$. For any $\mathrm{y} \in \mathbb{R}^{N}$ let $Y_{\mu}$ denote the $M \times N$ matrix with $\mathrm{y}$ in row $\mu$ and all other rows zero, $Y_{\mu} \equiv \hat{\mathbf{e}}_{\mu} \mathrm{y}^{\mathrm{t}}$. Note

$$
\left(E, X_{i}\right)_{i \leqslant N}=E^{\mathrm{t}} \mathbf{x}, \quad\left(E, Y_{\mu}\right)_{\mu \leqslant M}=E \mathbf{y} .
$$

Recalling (33) and (38), let $\boldsymbol{V}_{\mu} \equiv \hat{\mathbf{e}}_{\mu} \mathbf{v}^{\mathrm{t}}$, and define similarly $\tilde{V}_{\mu}$ and $W_{\mu}$.

\subsection{Orthogonalized TAP Equations}

Recall that $\left(\mathbf{r}^{(s)}\right)_{1 \leqslant s \leqslant t}$ is an orthonormal basis for the subspace $\operatorname{span}\left(\left(\mathbf{m}^{(s)}\right)_{1 \leqslant s \leqslant t-1}, \mathbf{m}\right)$, obtained by the change of coordinates (QR factorization) $\boldsymbol{M}=\boldsymbol{R} \boldsymbol{\Gamma}$ for $\boldsymbol{M}$ as in (31). Similarly, $\left(\mathbf{c}^{(s)}\right)_{1 \leqslant s \leqslant t}$ is an orthonormal basis for $\operatorname{span}\left(\left(\mathbf{n}^{(s)}\right)_{1 \leqslant s \leqslant t-1}, \mathbf{n}\right)$, obtained by the change of coordinates $N=C \Xi$ for $N$ as in (32). The constraints (56) and (57) can be rewritten as

$$
\begin{aligned}
\boldsymbol{E r}^{(s)} & =\mathbf{x}^{(s)} \text { for } 1 \leqslant s \leqslant t, \\
\boldsymbol{E}^{\mathrm{t}} \mathbf{c}^{(s)} & =\mathbf{y}^{(s)} \text { for } 1 \leqslant s \leqslant t,
\end{aligned}
$$

where $\mathbf{x}^{(s)}$ and $\mathbf{y}^{(s)}$ are defined by the same change of coordinates:

$$
\left(\begin{array}{c}
\mathbf{x}^{(t)} \\
\vdots \\
\mathbf{x}^{(1)}
\end{array}\right)=\left(\boldsymbol{\Gamma}^{-1}\right)^{\mathrm{t}}\left(\begin{array}{c}
{[\mathbf{h}+(1-q \cdot) \mathbf{n}] /(q \cdot)^{1 / 2}} \\
{\left[\mathbf{h}^{(t-1)}+b^{(t-1)} \mathbf{n}^{(t-2)}\right] /\left(q_{t-1}\right)^{1 / 2}} \\
\vdots \\
{\left[\mathbf{h}^{(1)}+b^{(1)} \mathbf{n}^{(0)}\right] /\left(q_{1}\right)^{1 / 2}}
\end{array}\right)
$$

and similarly

$$
\left(\begin{array}{c}
\mathbf{y}^{(t)} \\
\cdots \\
\mathbf{y}^{(1)}
\end{array}\right)=\left(\boldsymbol{\Gamma}^{-1}\right)^{\mathrm{t}}\left(\begin{array}{c}
{\left[\mathbf{H}^{(t+1)}+d^{(t)} \mathbf{m}^{(t)}\right] /\left(\psi_{\bullet}\right)^{1 / 2}} \\
{\left[\mathbf{H}^{(t)}+d^{(t-1)} \mathbf{m}^{(t-1)}\right] /\left(\psi_{t-1}\right)^{1 / 2}} \\
\vdots \\
{\left[\mathbf{H}^{(2)}+d^{(1)} \mathbf{m}^{(1)}\right] /\left(\psi_{1}\right)^{1 / 2}}
\end{array}\right) .
$$

Let $R_{\mu}^{(s)} \equiv \hat{\mathbf{e}}_{\mu}\left(\mathbf{r}^{(s)}\right)^{\mathrm{t}}$ and $C_{i}^{(s)} \equiv \mathbf{c}^{(s)}\left(\dot{\mathbf{e}}_{i}\right)^{\mathrm{t}}$, and define subspaces

$$
\begin{aligned}
& V_{R} \equiv \operatorname{span}\left(\left(\boldsymbol{R}_{\mu}^{(s)}\right)_{1 \leqslant s \leqslant t, \mu \leqslant M}\right), \\
& V_{C} \equiv \operatorname{span}\left(\left(C_{i}^{(s)}\right)_{1 \leqslant s \leqslant t, i \leqslant N}\right) .
\end{aligned}
$$

For $\mathbf{v}$ as given by (33), define $\boldsymbol{V}^{(s)} \equiv \mathbf{c}^{(s)} \mathbf{v}^{\mathrm{t}}$, and similarly $\tilde{\boldsymbol{V}}^{(s)}$ and $\boldsymbol{W}^{(s)}$. Observe that $\boldsymbol{V}^{(s)}, \tilde{\boldsymbol{V}}^{(s)}, \boldsymbol{W}^{(s)}$ are all orthogonal to $V_{R}$ and lie in $V_{C}$. For any $\boldsymbol{E}$ satisfying the column constraints (COL), we must have

$$
\begin{aligned}
& \left(\boldsymbol{E}, \boldsymbol{V}^{(s)}\right)=\left(\mathbf{c}^{(s)}\right)^{\mathrm{t}} \boldsymbol{E} \mathbf{v}=\left(\mathbf{y}^{(s)}, \mathbf{v}\right) \text { for } 1 \leqslant s \leqslant t, \\
& \left(\boldsymbol{E}, \tilde{\boldsymbol{V}}^{(s)}\right)=\left(\mathbf{c}^{(s)}\right)^{\mathrm{t}} \boldsymbol{E} \tilde{\mathbf{v}}=\left(\mathbf{y}^{(s)}, \tilde{\mathbf{v}}\right) \text { for } 1 \leqslant s \leqslant t .
\end{aligned}
$$

We refer to $\left(\mathrm{ADM}_{1}\right)$ and $\left(\mathrm{ADM}_{2}\right)$ as the admissibility constraints. Define the following subspaces of $\mathbb{R}^{M \times N}$ :

$$
\begin{aligned}
& V_{A_{1}}=\operatorname{span}\left(\left(\boldsymbol{V}^{(s)}\right)_{1 \leqslant s \leqslant t}\right), \\
& V_{P_{1}}=\operatorname{span}\left(\left(\boldsymbol{V}_{\mu}\right)_{\mu \leqslant M}\right)=V_{A_{1}} \oplus V_{Q_{1}}, \\
& V_{A_{2}}=\operatorname{span}\left(\left(\tilde{\boldsymbol{V}}^{(s)}\right)_{1 \leqslant s \leqslant t}\right), \\
& V_{P_{2}}=\operatorname{span}\left(\left(\tilde{\boldsymbol{V}}_{\mu}\right)_{\mu \leqslant M}\right)=V_{A_{2}} \oplus V_{Q_{2}} .
\end{aligned}
$$

We use $\ominus$ to refer to the orthogonal sum of two subspaces, so $V_{Q_{1}}$ is by definition the orthogonal complement of $V_{A_{1}}$ inside $V_{P_{1}}$. For any subspaces $V_{X}, V_{Y}$ we abbreviate their direct (not necessarily orthogonal) sum as

$$
V_{X Y} \equiv V_{X} \oplus V_{Y}
$$

Let $V_{A} \equiv V_{A_{1} A_{2}}, V_{Q} \equiv V_{Q_{1} Q_{2}}$, and $V_{P} \equiv V_{P_{1} P_{2}}=V_{A Q}$.

LEMMA 3.1 (subSPACE DECOMPOSITIONS). Note from the definitions that $V_{A_{1}} \subseteq V_{A} \subseteq V_{R C}$. Let $V_{D_{1}}$ and $V_{D}$ be defined by the orthogonal decomposition relations

$$
V_{R} \oplus V_{A_{1}} \oplus V_{D_{1}}=V_{R C}=V_{R} \oplus V_{A} \oplus V_{D} .
$$

We then have the additional relations

$$
V_{R} \oplus V_{P_{1}} \oplus V_{D_{1}}=V_{R C P_{1}}, \quad V_{R} \oplus V_{P} \oplus V_{D}=V_{R C P} .
$$


Proof. We prove the claim for $V_{D}$ only, as the claim for $V_{D_{1}}$ follows by a similar argument. For each $s$ and $i$, let $A_{i}^{(s)}$ be the projection of $C_{i}^{(s)}$ onto the orthogonal complement of $V_{R} \oplus V_{A}$,

$$
\begin{aligned}
A_{i}^{(s)}=C_{i}^{(s)} & -\sum_{r=1}^{t} \sum_{\mu=1}^{M}\left(C_{i}^{(s)}, \boldsymbol{R}_{\mu}^{(r)}\right) \boldsymbol{R}_{\mu}^{(r)} \\
& -\sum_{r=1}^{t}\left\{\left(C_{i}^{(s)}, V^{(r)}\right) V^{(r)}+\left(C_{i}^{(s)}, W^{(r)}\right) W^{(r)}\right\} .
\end{aligned}
$$

One can check that $\left(C_{i}^{(s)}, V^{(r)}\right)=0$ for $r \neq s$, while

$$
\begin{aligned}
\left(C_{i}^{(s)}, V^{(s)}\right) V^{(s)} & =\sum_{j \leqslant N} v_{j}\left(C_{i}^{(s)}, C_{j}^{(s)}\right) V^{(s)} \\
& =v_{i} \boldsymbol{V}^{(s)}=\sum_{\mu=1}^{M}\left(C_{i}^{(s)}, V_{\mu}\right) \boldsymbol{V}_{\mu}
\end{aligned}
$$

(and similarly with $\boldsymbol{W}$ in place of $\boldsymbol{V}$ ). It follows that

$$
\begin{aligned}
A_{i}^{(s)}=C_{i}^{(s)} & -\sum_{\mu=1}^{M} \sum_{r=1}^{t}\left(C_{i}^{(s)}, R_{\mu}^{(r)}\right) R_{\mu}^{(r)} \\
& -\sum_{\mu=1}^{M}\left\{\sum_{r=1}^{t}\left[\left(C_{i}^{(s)}, V_{\mu}\right) V_{\mu}+\left(C_{i}^{(s)}, W_{\mu}\right) W_{\mu}\right]\right\},
\end{aligned}
$$

from which we immediately see that each $A_{i}^{(s)}$ is in fact orthogonal to $V_{R} \oplus V_{P}$. The space $V_{D}$ is spanned by the $A_{i}^{(s)}$, and it follows that $V_{R} \oplus V_{P} \oplus V_{D}=V_{R C P}$.

\subsection{Change of Measure}

Recall from Remark 2.8 that $E$ is a standard gaussian random variable in $\mathbb{R}^{M \times N}$ which is independent of the background $\sigma$-field $\mathscr{F}$ bg . Let $\mathbb{P}$ denote the law of $\boldsymbol{E}$ conditional on $\mathscr{F}$ bg (which, by independence, is the same as the unconditional law). We will make calculations under a tilted measure $\check{\mathbb{P}}$, defined by

$$
\frac{d \check{\mathbb{P}}}{d \mathbb{P}}=\exp \left\{(\boldsymbol{E}, \boldsymbol{T})-\frac{\|\boldsymbol{T}\|^{2}}{2}\right\}
$$

for $T$ to be specified below. Let $\check{p}$ denote the density of $\boldsymbol{E}$ under $\check{\mathbb{P}}$. We indicate projections of $\boldsymbol{E}$ onto subspaces by subscripts, e.g. the projection of $E$ onto $V_{R}$ is denoted $E_{R}$. We shall prove the following:

Proposition 3.2 (Change of measure formula). For any $\boldsymbol{E}_{R P^{-}}$ measurable event $B$, and for any $T \in V_{R C}$,

$$
\mathbb{P}\left(B \mid E_{R C}\right)=\frac{\check{\mathbb{P}}\left(B \mid E_{R}\right) \check{p}_{A \mid R B}\left(E_{A} \mid E_{R}\right)}{\check{p}_{A}\left(E_{A}\right)}
$$

where $\check{p}_{A \mid R B}\left(E_{A} \mid E_{R}\right)$ is the density of $E_{A}$ under the conditional law $\check{\mathbb{P}}\left(\cdot \mid E_{R}=E_{R}, E_{R P} \in B\right)$. Likewise, for any $E_{R P_{1}}-$ measurable event $B_{1}$,

$$
\mathbb{P}\left(B_{1} \mid E_{R C}\right)=\frac{\check{\mathbb{P}}\left(B_{1} \mid E_{R}\right) \check{p}_{A_{1} \mid R B_{1}}\left(E_{A_{1}} \mid E_{R}\right)}{\check{p}_{A_{1}}\left(E_{A_{1}}\right)}
$$

where $\check{p}_{A_{1} \mid R B_{1}}\left(E_{A_{1}} \mid E_{R}\right)$ is the density of $E_{A_{1}}$ under the conditional law $\check{\mathbb{P}}\left(\cdot \mid E_{R}=E_{R}, E_{R P_{1}} \in B_{1}\right)$.

Proof. We prove $(S \mid C R)$ only, as $\left(\mathrm{S}_{1} \mid \mathrm{CR}\right)$ follows similarly. Write $p$ and $\check{p}$ for densities under $\mathbb{P}$ and $\check{\mathbb{P}}$ respectively. From Lemma 3.1 we have the orthogonal decompositions

$$
\begin{aligned}
& V_{R} \oplus V_{A} \oplus V_{D}=V_{R C}, \\
& V_{R} \oplus V_{P} \oplus V_{D}=V_{R C P} .
\end{aligned}
$$

Recall also that $V_{P}=V_{A Q}$. Therefore the left-hand side of $(\mathrm{S} \mid \mathrm{CR})$ can be written as

$$
\mathbb{P}\left(B \mid E_{R C}\right)=\frac{1}{p_{R A D}\left(E_{R A D}\right)} \int 1\left\{E_{R A Q} \in B\right\} p_{R A Q D}\left(E_{R A Q D}\right) d E_{Q} .
$$

The right-hand side above is unaffected if we replace $p$ with $\check{p}$, because $T \in V_{R C}$ and so the Radon-Nikodym derivative (61) takes the same fixed value in both numerator and denominator. After replacing $p$ with $\check{p}$, we apply the orthogonality result of Lemma 3.1 to simplify

$$
\begin{aligned}
\mathbb{P}\left(B \mid E_{R C}\right) & =\frac{\check{\mathbb{P}}\left(B \mid E_{R}\right)}{\check{p}_{A}\left(E_{A}\right)} \int \frac{1\left\{E_{R A Q} \in B\right\} \check{p}_{A Q}\left(E_{A Q}\right)}{\check{\mathbb{P}}\left(B \mid E_{R}\right)} d E_{Q} \\
& =\frac{\check{\mathbb{P}}\left(B \mid E_{R}\right) \check{p}_{A \mid R B}\left(E_{A} \mid E_{R}\right)}{\check{p}_{A}\left(E_{A}\right)},
\end{aligned}
$$

where the last equality holds by definition of $\check{p}_{A \mid R B}\left(E_{A} \mid E_{R}\right)$. This proves the formula $(\mathrm{S} \mid \mathrm{CR})$.

For $E \in \mathbb{R}^{M \times N}$ satisfying the equations (ROW) and (COL) (and hence also satisfying $\left(\mathrm{ADM}_{1}\right)$ and $\left.\left(\mathrm{ADM}_{2}\right)\right)$ we will have the projections $E_{R}$, $E_{C}, E_{A}, E_{D}$ constrained to fixed values, hereafter denoted $\bar{E}_{R}, \bar{E}_{C}$, $\bar{E}_{A}, \bar{E}_{D}$ : for instance,

$$
\bar{E}_{A}=\sum_{s=1}^{t}\left\{\left(\mathbf{v}, \mathbf{y}^{(s)}\right) \boldsymbol{V}^{(s)}+\left(\mathbf{w}, \mathbf{y}^{(s)}\right) \boldsymbol{W}^{(s)}\right\} .
$$

Recalling Definition 2.6, we fix a pair

$$
(J, K) \in \mathbb{H}(\lambda) \equiv \mathbb{H}_{M, N_{\text {all }}, \delta, t}(\lambda),
$$

and calculate the terms of Proposition 3.2 with $E_{R C}=\bar{E}_{R C}$ and

$$
\begin{aligned}
B_{1} & =S_{J, \epsilon} \equiv S_{J, \delta, t, \epsilon}, \\
B & =\left\{B_{1}, S_{K}\right\} \equiv\left\{B_{1}, S_{K, \delta, t}\right\} .
\end{aligned}
$$

This calculation occupies the majority of the full version of this paper, which is available online.

\section{ACKNOWLEDGMENTS}

We are extremely grateful to Andrea Montanari who generously discussed this problem with us on many occasions, and shared ideas that became essential to the proof. We also wish to thank Erwin Bolthausen for sharing with us the manuscripts of his related work. Riccardo Zecchina, Carlo Baldassi, and Lenka Zdeborová introduced us to this problem, and we are grateful for their encouragement to work on it. The perceptron model was also brought up at an American Institute of Mathematics workshop in June 2017, and we thank the other participants in the discussions there: Dimitris Achlioptas, Nick Cook, Reza Gheissari, Aukosh Jagannath, Florent Krzakala, Will Perkins, Eliran Subag, Yumeng Zhang. We also thank Florent Krzakala for helpful conversations on the TAP free energy. Finally, it is a pleasure to acknowledge the hospitality of our colleagues at the National University of Singapore and at the Centre de Recherches Mathématiques, where parts of this work were completed. J.D. is supported by NSF grant DMS-1757479 and an Alfred Sloan fellowship. N.S. is supported by NSF grant DMS-1752728. 


\section{REFERENCES}

[1] Carlo Baldassi, Christian Borgs, Jennifer T Chayes, Alessandro Ingrosso, Carlo Lucibello, Luca Saglietti, and Riccardo Zecchina. 2016. Unreasonable effectiveness of learning neural networks: From accessible states and robust ensembles to basic algorithmic schemes. Proc. Natl. Acad. Sci. 113, 48 (2016), E7655-E7662

[2] Carlo Baldassi, Alessandro Ingrosso, Carlo Lucibello, Luca Saglietti, and Riccardo Zecchina. 2016. Local entropy as a measure for sampling solutions in constraint satisfaction problems. F. Stat. Mech. 2016, 2 (2016), 023301.

[3] Mohsen Bayati and Andrea Montanari. 2011. The dynamics of message passing on dense graphs, with applications to compressed sensing. IEEE Trans. Inform. Theory 57, 2 (2011), 764-785. https://doi.org/10.1109/TIT.2010.2094817

[4] Mohsen Bayati and Andrea Montanari. 2012. The LASSO risk for Gaussian matrices. IEEE Trans. Inform. Theory 58, 4 (2012), 1997-2017. https://doi.org/10.1109/TIT.2011.2174612

[5] Erwin Bolthausen. 2014. An iterative construction of solutions of the TAP equations for the Sherrington-Kirkpatrick model. Comm. Math. Phys. 325, 1 (2014), 333-366. https://doi.org/10.1007/s00220-013-1862-3

[6] Erwin Bolthausen. 2018. A Morita type proof of the replica-symmetric formula for SK. arXiv:1809.07972.

[7] David Donoho and Andrea Montanari. 2016. High dimensional robust M-estimation: asymptotic variance via approximate message passing. Probab. Theory Related Fields 166, 3-4 (2016), 935-969.

https://doi.org/10.1007/s00440-015-0675-z

[8] Zhou Fan, Song Mei, and Andrea Montanari. 2018. TAP free energy, spin glasses, and variational inference. arXiv:1808.07890.

[9] Silvio Franz and Giorgio Parisi. 2016. The simplest model of jamming. 7. Phys. A 49, 14 (2016), 145001.

[10] Silvio Franz, Giorgio Parisi, Maxime Sevelev, Pierfrancesco Urbani, and Francesco Zamponi. 2017. Universality of the SAT-UNSAT (jamming) threshold in non-convex continuous constraint satisfaction problems. SciPost Physics 2, 3 (2017), 019.

[11] Elizabeth Gardner. 1987. Maximum storage capacity in neural networks. Europhys. Lett. 4,4 (1987), 481.

[12] Elizabeth Gardner. 1988. The space of interactions in neural network models. $\mathcal{F}$. Phys. A 21, 1 (1988), 257.

[13] E Gardner and B Derrida. 1988. Optimal storage properties of neural network models. F. Phys. A 21, 1 (1988), 271.
[14] Francesco Guerra and Fabio Lucio Toninelli. 2002. The thermodynamic limit in mean field spin glass models. Comm. Math. Phys. 230, 1 (2002), 71-79. https://doi.org/10.1007/s00220-002-0699-y

[15] Jeong Han Kim and James R. Roche. 1998. Covering cubes by random half cubes, with applications to binary neural networks. F. Comput. System Sci. 56, 2 (1998), 223-252. https://doi.org/10.1006/jcss.1997.1560

[16] Werner Krauth and Marc Mézard. 1989. Storage capacity of memory networks with binary couplings. F. Physique 50, 20 (1989), 3057-3066.

[17] Marc Mézard. 1989. The space of interactions in neural networks: Gardner's computation with the cavity method. F. Phys. A 22, 12 (1989), 2181.

[18] Dmitry Panchenko. 2013. The Sherrington-Kirkpatrick model. Springer, New York. xii+156 pages. https://doi.org/10.1007/978-1-4614-6289-7

[19] Cynthia Rush and Ramji Venkataramanan. 2018. Finite sample analysis of approximate message passing algorithms. IEEE Trans. Inform. Theory 64, 11 (2018), 7264-7286.

[20] Mariya Shcherbina and Brunello Tirozzi. 2003. Rigorous solution of the Gardner problem. Comm. Math. Phys. 234, 3 (2003), 383-422. https://doi.org/10.1007/s00220-002-0783-3

[21] David Sherrington and Scott Kirkpatrick. 1975. Solvable model of a spin-glass. Phys. Rev. Lett. 35, 26 (1975), 1792.

[22] Mihailo Stojnic. 2013. Another look at the Gardner problem. arXiv:1306.3979.

[23] Mihailo Stojnic. 2013. Discrete perceptrons. arXiv:1306.4375.

[24] Michel Talagrand. 1999. Intersecting random half cubes. Random Structures Algorithms 15, 3-4 (1999), 436-449. https://doi.org/10.1002/(SICI)1098-2418(199910/12)15:3/4<436:: AID-RSA11 > 3.0.CO;2-5

[25] Michel Talagrand. 2000. Intersecting random half-spaces: toward the Gardner-Derrida formula. Ann. Probab. 28, 2 (2000), 725-758. https://doi.org/10.1214/aop/1019160259

[26] Michel Talagrand. 2002. On the high temperature phase of the Sherrington-Kirkpatrick model. Ann. Probab. 30, 1 (2002), 364-381. https://doi.org/10.1214/aop/1020107771

[27] Michel Talagrand. 2006. The Parisi formula. Ann. of Math. (2) 163, 1 (2006), 221-263. https://doi.org/10.4007/annals.2006.163.221

[28] Michel Talagrand. 2011. Mean field models for spin glasses. Volume I. Vol. 54 Springer-Verlag, Berlin. xviii+485 pages. https://doi.org/10.1007/978-3-642-15202-3 Basic examples.

[29] David J. Thouless, Philip W. Anderson, and Robert G. Palmer. 1977. Solution of 'Solvable model of a spin glass'. Philos. Mag. 35, 3 (1977), 593-601. 